\title{
Target Localization by Resolving the Time Synchronization Problem in Bistatic Radar Systems Using Space Fast-Time Adaptive Processor
}

\author{
D. Madurasinghe and A. P. Shaw \\ Electronic Warfare and Radar Division, Defence Science and Technology Organisation, P.O. Box 1600, Edinburgh, SA 5111, Australia
}

Correspondence should be addressed to D. Madurasinghe, dan.madurasinghe@dsto.defence.gov.au

Received 30 September 2008; Accepted 26 January 2009

Recommended by Magnus Jansson

The proposed technique allows the radar receiver to accurately estimate the range of a large number of targets using a transmitter of opportunity as long as the location of the transmitter is known. The technique does not depend on the use of communication satellites or GPS systems, instead it relies on the availability of the direct transmit copy of the signal from the transmitter and the reflected paths off the various targets. An array-based space-fast time adaptive processor is implemented in order to estimate the path difference between the direct signal and the delayed signal, which bounces off the target. This procedure allows us to estimate the target distance as well as bearing.

Copyright ( 2009 D. Madurasinghe and A. P. Shaw. This is an open access article distributed under the Creative Commons Attribution License, which permits unrestricted use, distribution, and reproduction in any medium, provided the original work is properly cited.

\section{Introduction}

Bistatic radar systems are gaining more and more interest over the past two decades due to the freedom and flexibility it offers in deploying transmitters and receivers. Other advantages include the ability to use inexpensive receive modules, the use of continuous wave signals, the use of transmitters of opportunity, lower maintenance cost, operation without frequency clearance (if using third party transmitters), covert operation of the receiver, increase resilience to electrometric countermeasures, ability to hide the receiver location and the waveform being used, and huge enhancement of the target radar cross-section due to geometrical effects. However, several disadvantages include the system complexity, cost of providing communication between sites, lack of any control over the transmitter (if using third party transmitters), and reduced low-level coverage due to the need for line-of-sight from several locations.

Passive radar systems (also referred to as passive coherent location and passive covert radar) encompass a class of radar systems that detect and track objects by processing reflections from noncooperative sources of illumination in the environment, such as commercial broadcast and communications signals. It is a specific case of bistatic radar that exploites cooperative and noncooperative radar transmitters. References [1-5] are some of the examples.

In bistatic radar systems, the time synchronization is one of the most important key technology areas. This is necessary to maintain bistatic phase coherency between the transmitter and the receiver. This is the main factor that may severely limit the radar performance. Because of the separation between the transmitter and the receiver, one needs to maintain the synchronization of receive and transmit signals, that is, accurate phase information, transmit time. Transmitter geolocation needs to be conveyed about the transmitter itself and the transmitted pulse to the receiver to reconstitute a phase coherent image at the receiver. For bistatic radar usually two or more separate local oscillators (LO), one in the transmitters and one in each of the receivers, need to be synchronized. In a monostatic configuration, the same LO is shared physically by both the transmitter and the receiver avoiding the need for synchronization. In bistatic configurations, the transmitter-related information is delivered by a separate data link between the transmitter and the receiver. Such a data link is highly probable for failures and demand additional hardware complexity. Other 
approaches include the use of GPS systems that may allow us to synchronize the time over a reasonably long period with a time difference of less than 1 nanosecond. This topic has been discussed widely in the existing literature by various authors and various improved methods are also available. References [6-9] are some of the examples. In this study we propose an innovative approach to locate the targets without the aid of the communication satellites or the GPS systems. Under the proposed technique, one does not need to maintain any form of synchronization between transmitter and receiver, in respect of, instant of pulse transmission and transmit signal phase.

This study introduces a technique to resolve the synchronization problem related to bistatic radar by using a new and emerging class of signal processing technique that may be referred to as space fast-time adaptive processing (SFTAP). The SFTAP is conventionally applied to null mainlobe interferers using an array of receivers in a monostatic configuration [10-15]. In a conventional space fast-time adaptive processor one blindly stacks a large number of consecutive range cell returns to form a space fast-time adaptive processor expecting that the process would null the interference signal (commonly known as the mainlobe signal) due to the presence of its delayed copies known as terrain scattered interference paths. Recent advances in this type of signal processing have led to the introduction of a processor known as the Terrain Scattered Interference (TSI) finder [14], the function of which is to avoid the stacking of a large number of range cells blindly, instead it leads the SFTAP processor to the correct range cell position to form the space fast-time data snap. The TSI finder basically identifies all the delayed copies of the signal of interest, which include the multipath bounces off various other targets and the ground. This is achieved by forming a space fast-time beam in the direction of the signal of interest, or in our case the transmitter, by assuming the bearing of the transmitter is precisely known. Such a beam is able to null all other existing sidelobe arrivals, which are known as interferers or jammers, which are uncorrelated with the signal of interest. The objective of the beam is to identify all the sidelobe arrivals which are delayed versions of the look direction signal.

An application of this theory would be the detection of airborne targets in a maritime environment where the transmitter is placed several kilometers away from the maritime platform in a known position (or the position of a moving transmitter location is accurately known to the receiver system in order to form the space-time beam at any given time). Another important application would be to detect high altitude or space-based targets, such as intercontinental ballistic missiles, using a bistatic arrangement where a series of transmitters and receivers can be geographically distributed to achieve the best possible results. In such a scenario, one would locate all the transmitters in high altitude locations (mountains), where receivers can receive direct signal (which can be a random continuous waveform) from all or most of the transmitters in order to track each of the multipath signals (target reflections) originating due to the known transmitters. The proposed

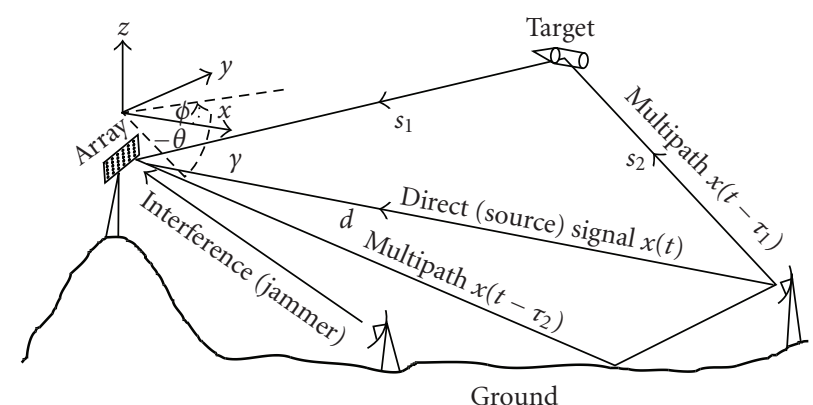

Figure 1: Transmitter and receiver arrangements with an airborne target, a jammer, and one transmitter.

algorithm will identify each multipath with its associated direct transmit signal, by forming a space fast-time beam in the direction of each known transmitter.

We aim to solve the problem by locking the radar receiver in the direction of a known transmitter at a known bearing and distance (usually a third party transmitter in the line-of-sight). The objective is to receive its direct signal by forming a beam in the direction of the transmitter (a space-time beam), which allows us to effectively form a secondary search beam for arrival of the same stream of data (with a delay) due to reflections off the targets and the ground (these beams are formed simultaneously). Such delayed versions usually have a different bearing and a fixed delay factor during the integration period. In this study these are termed as multipath arrivals of the main beam signal (or in the case of ground reflections they are termed as TSI arrivals). Once this knowledge is established, for every multipath or TSI arrival, one can estimate the location of the reflection point via triangulation. While some points are identified as targets some may correspond to ground reflections. Reflection points which vary over time may be classified as moving targets, at a postprocessing stage.

In this study, first we formulate the problem (Section 2), and then in Section 3 we discuss the properties of the original TSI finder. In Section 4 we introduce the second processor (a postprocessor) to identify all target bearings that may include all the bounced rays off the moving targets as well as stationary targets (ground reflections), by forming a beam in the desired direction which in this case is the transmitter direction. In order to achieve this, we introduce an innovative multipath bearing estimator using two very different optimization approaches. Both solutions are discussed in detail as potential solutions to the multipath bearing estimation problem. Section 5 briefly presents the formula for estimating the target location. Finally in Section 6 we carry out a simulation study to demonstrate bistatic scenarios including multiple air target detection using a known transmitter in a known direction, which transmits a random continuous wave signal.

\section{Formulation}

Suppose we have an $N$-channel radar receiver (Figure 1) whose $N \times 1$ steering manifold is represented by $\mathbf{s}(\phi, \theta)$, 
where $\phi$ is the azimuth angle, $\theta$ is the elevation angle, $\mathbf{s}(\phi, \theta)^{H} \mathbf{s}(\phi, \theta)=N$, and the superscript $H$ denotes the Hermitian transpose. The $t$ th range gate, $N \times 1$ measured signal $\mathbf{x}(t) \quad(t$ is also the fast-time scale or an instant of sampling in fast-time) can be written as

$$
\begin{aligned}
\mathbf{x}(t)= & j_{1}(t) \mathbf{s}\left(\phi_{1}, \theta_{1}\right)+j_{2}(t) \mathbf{s}\left(\phi_{2}, \theta_{2}\right) \\
& +\sum_{m=1}^{a_{1}} \beta_{1, m} j_{1}\left(t-n_{1, m}\right) \mathbf{s}\left(\phi_{1, m}, \theta_{1, m}\right) \\
& +\sum_{m=1}^{a_{2}} \beta_{2, m} j_{2}\left(t-n_{2, m}\right) \mathbf{s}\left(\phi_{2, m}, \theta_{2, m}\right)+\boldsymbol{\varepsilon},
\end{aligned}
$$

where $j_{1}(t), j_{2}(t)$ represent a series of complex random amplitudes corresponding to two far field sources, with the directions of arrival pairs, $\left(\phi_{1}, \theta_{1}\right)$ and $\left(\phi_{2}, \theta_{2}\right)$, respectively. The third term represents Scattered Interference (in our case, multipath bounces) paths off the first source with time lags (path lags) $n_{1,1}, n_{1,2}, n_{1,3}, \ldots, n_{1, a_{1}}$, the scattering coefficients $\left|\beta_{1, m}\right|^{2}<1, m=1,2, \ldots, a_{1}$, and the associated direction of arrival pairs $\left(\phi_{1, m}, \theta_{1, m}\right)\left(m=1,2, \ldots, a_{1}\right)$. The fourth term is the multipaths off the second source with path delays $n_{2,1}, n_{2,2}, n_{2,3}, \ldots, n_{2, a_{2}}$, the scattering coefficients $\left|\beta_{2, m}\right|^{2}<1, m=1,2, \ldots, a_{2}$, and the associated direction of arrivals $\left(\phi_{2, m}, \theta_{2, m}\right)\left(m=1,2, \ldots, a_{2}\right)$. More sources and multiple paths from each source are accepted in general, but for the sake of brevity, we represent one of each and $\boldsymbol{\varepsilon}$ represents the $N \times 1$ white noise component. In this study we consider the clutter-free case. Furthermore, we assume that $\rho_{k}^{2}=E\left\{\left|j_{k}(t)\right|^{2}\right\}(k=1,2, \ldots)$ are the power levels of each source, and $\left|\beta_{k, m}\right|^{2} \rho_{k}^{2}(m=1,2, \ldots)$ represent the multipath power levels associated with each bounce from the $k$ th source, where $E\{\cdot\}$ denotes the expectation operator with respect to the variable $t$. Throughout the analysis we assume that we are interested only in the source powers (as potential transmit sources) that are above the channel noise power, that is, $\operatorname{snr}_{k}=\rho_{k}^{2} / \sigma_{n}^{2}>1, k=1,2, \ldots$, $E\left\{\boldsymbol{\varepsilon} \varepsilon^{H}\right\}=\sigma_{n}^{2} \mathbf{I}_{N}$, where $\operatorname{snr}_{k}$ is the transmit source power to noise power ratio per channel, $\sigma_{n}^{2}$ is the white noise power present in any channel, and $\mathbf{I}_{N}$ is the unit identity matrix (the effect of $\operatorname{snr}_{k}=\rho_{k}^{2} / \sigma_{n}^{2}<1, k=1,2, \ldots$, is discussed in the simulation section). Without loss of generality we use the notations $\mathbf{s}_{1}$ and $\mathbf{s}_{2}$ to represent $\mathbf{s}\left(\phi_{1}, \theta_{1}\right)$ and $\mathbf{s}\left(\phi_{2}, \theta_{2}\right)$, respectively, but the steering vectors associated with multipath arrivals are represented by two subscript notations $\mathbf{s}_{1, m}=\mathbf{s}\left(\phi_{1, m}, \theta_{1, m}\right)\left(m=1,2, \ldots, a_{1}\right), \mathbf{s}_{2, m}=$ $\mathbf{s}\left(\phi_{2, m}, \theta_{2, m}\right)\left(m=1,2, \ldots, a_{2}\right)$, and so on. Furthermore it is assumed that $E\left\{j_{k}(t+l) j_{k}(t+m)^{*}\right\}=\rho_{k}^{2} \delta(l-m)(k=$ $1,2, \ldots)$, where $*$ denotes the complex conjugate operation. This last assumption restricts the application of this theory to noise-like sources that are essentially continuous over the period of examination.

\section{Multipath Lag Finder}

3.1. Multipath Lag versus Power Spectrum. This section looks at a technique that will identify each source (given the source direction) and its associated multipath arrivals (if present).
Here we assume that the radar has been able to identify the desired source as the suitable transmitter (i.e., $\rho_{k}^{2} / \sigma_{n}^{2}>1$ ) and we would like to identify all its associated multipaths. The formal use of the multipaths (known as Terrain Scattered Interference paths or TSI) is very well known in literature under the topic mainlobe jammer nulling [10-14]. However, the use of the multipath in this study is restricted to the bounces off the airborne targets (reflections off the ground are discarded as discussed later). Throughout this study we assume that the first source is our desired transmit source with the known bearing. The array's $N \times N$ spatial covariance matrix has the following structure (for the case where two sources and one multipath off each source is present):

$$
\begin{aligned}
\mathbf{R}_{x}= & \rho_{1}^{2} \mathbf{s}_{1} \mathbf{s}_{1}^{H}+\rho_{2}^{2} \mathbf{s}_{2} \mathbf{s}_{2}^{H}+\rho_{1}^{2}\left|\beta_{1,1}\right|^{2} \mathbf{s}_{1,1} \mathbf{s}_{1,1}^{H} \\
& +\rho_{2}^{2}\left|\beta_{2,1}\right|^{2} \mathbf{s}_{2,1} \mathbf{s}_{2,1}^{H}+\sigma_{n}^{2} \mathbf{I}_{N} .
\end{aligned}
$$

Suppose now we compute the space fast-time covariance $\mathbf{R}_{2}$ of size $2 \mathrm{~N} \times 2 \mathrm{~N}$ corresponding to an arbitrarily chosen fast-time lag $n$, then we have

$$
\begin{array}{r}
\mathbf{R}_{2}=E\left\{\mathbf{X}_{n}(t) \mathbf{X}_{n}(t)^{H}\right\}=\left(\begin{array}{cc}
\mathbf{R}_{x} & \mathbf{O}_{N \times N} \\
\mathbf{O}_{N \times N} & \mathbf{R}_{x}
\end{array}\right) \\
\text { for } n \neq n_{1, m} \text { or } n_{2, m} m=1,2, \ldots,
\end{array}
$$

where $\mathbf{X}_{n}(t)=\left(\mathbf{x}(t)^{T}, \mathbf{x}(t+n)^{T}\right)^{T}$ is termed as the $2 N \times 1$ space fast-time snapshot for the selected lag $n$ and $\mathbf{O}_{N \times N}$ is the $N \times N$ matrix with zero entries. However if $n=$ $n_{1, m}$ or $n_{2, m}$ for some $m$ then we have (say $n=n_{1,1}$ as an example)

$$
\begin{aligned}
\mathbf{X}_{n_{1}}(t)= & \left(\begin{array}{c}
\mathbf{x}(t) \\
\mathbf{x}\left(t+n_{1,1}\right)
\end{array}\right) \\
= & j_{1}(t)\left(\begin{array}{c}
\mathbf{s}_{1} \\
\beta_{1,1} \mathbf{s}_{1,1}
\end{array}\right)+j_{2}(t)\left(\begin{array}{c}
\mathbf{s}_{2} \\
\mathbf{o}_{N \times 1}
\end{array}\right) \\
& +\beta_{1,1} j_{1}\left(t-n_{1,1}\right)\left(\begin{array}{c}
\mathbf{s}_{1,1} \\
\mathbf{o}_{N \times 1}
\end{array}\right)+\beta_{2,1} j_{2}\left(t-n_{2,1}\right)\left(\begin{array}{c}
\mathbf{s}_{2,1} \\
\mathbf{o}_{N \times 1}
\end{array}\right) \\
& +j_{1}\left(t+n_{1,1}\right)\left(\begin{array}{c}
\mathbf{o}_{N \times 1} \\
\mathbf{s}_{1}
\end{array}\right)+j_{2}\left(t+n_{1,1}\right)\left(\begin{array}{c}
\mathbf{o}_{N \times 1} \\
\mathbf{s}_{2}
\end{array}\right) \\
& +\beta_{2,1} j_{2}\left(t-n_{2,1}+n_{1,1}\right)\left(\begin{array}{c}
\mathbf{o}_{N \times 1} \\
\mathbf{s}_{2,1}
\end{array}\right)+\left(\begin{array}{c}
\boldsymbol{\varepsilon}_{1} \\
\boldsymbol{\varepsilon}_{2}
\end{array}\right),
\end{aligned}
$$

where $\varepsilon_{1}$ and $\varepsilon_{2}$ represent two independent measurements of the white noise component, and $\mathbf{o}_{N \times 1}$ is the $N \times 1$ column of zeros. In this case the space fast-time covariance matrix is given by

$$
\mathbf{R}_{2}=\left(\begin{array}{cc}
\mathbf{R}_{x} & \mathbf{Q}^{H} \\
\mathbf{Q} & \mathbf{R}_{x}
\end{array}\right),
$$

where $\mathbf{Q}=\rho_{1}^{2} \beta_{1,1} \mathbf{s}_{1,1} \mathbf{s}_{1}^{H}$. 
It is important to note that we assume that $n_{1, m}$ ( $m=$ $1,2, \ldots)$ represent digitized sample values of the fast-time variable $t$ and the reflected path is an integer-valued delay of the direct path. If this assumption is not satisfied, one would not achieve a perfect decorrelation, resulting in a nonzero off diagonal term in (5) and a clear distinction between (4) and (5) would not be possible. The existence of the delayed value of the term $\mathbf{Q}$ can be made equal to zero, or not by suitably choosing a delay value for $n$ when forming the space-time covariance matrix. However, $\mathbf{Q}$ is a matrix and as a result one must consider its determinant value in order to differentiate the two cases in (4) and (5). After extensive analysis, one may find the signal processing gain is not acceptable for this choice. A more physically meaningful measure would be to consider its contribution to the overall processor output power (when minimized with respect to the look direction constraint). Depending on whether the power contribution is zero or not we have the situation described in (4) or (5) clearly identified under the above assumptions. The scaled measure was introduced as the TSI finder [14], which is a function of the chosen delay value $n$, must represent the scaled version of the contribution due to the presence of $\mathbf{Q}$ at the total output power. Even though one can come up with many variations of the TSI finder based on the same principle, the one expressed in this study is tested and verified to have high signal processing gain as seen later (the performance degradation of the finder spectrum when the path delay is not an integer multiple of the range resolution is discussed in the simulation section). Now suppose the direction of arrival of the mainlobe source (transmitter) to be $\left(\phi_{1}, \theta_{1}\right)$, the first objective is to find all its associated path delays, which may be of low power. This is carried out by the lag finder in the lag domain by searching over all possible lag values while the look direction is fixed at the desired source direction $\left(\phi_{1}, \theta_{1}\right)$. This is given by the spectrum

$$
T_{s}(n)=\left\{\frac{1}{P_{\text {out }}\left(\mathbf{s}_{1}^{H} \mathbf{R}_{x}^{-1} \mathbf{s}_{1}\right)}-1\right\},
$$

where $P_{\text {out }}=\mathbf{w}^{H} \mathbf{R}_{2} \mathbf{w}, \mathbf{w}$ is the $2 \mathrm{~N} \times 1$ space fast-time weights vector which minimizes the power while looking into the direction of the source of interest (transmitter) subject to the constraints: $\mathbf{w}^{H} \mathbf{s}_{A}=1$ and $\mathbf{w}^{H} \boldsymbol{s}_{B}=0$, where $\mathbf{s}_{A}=$ $\left(\mathbf{s}_{1}^{T}, \mathbf{o}_{N \times 1}^{T},\right)^{T} \mathbf{s}_{B}=\left(\mathbf{o}_{N \times 1}^{T}, \mathbf{s}_{1}^{T}\right)^{T}$. The solution $\mathbf{w}$ for each lag is given by $\mathbf{w}=\lambda \mathbf{R}_{2}^{-1} \mathbf{s}_{A}+\mu \mathbf{R}_{2}^{-1} \mathbf{s}_{B}$, where the parameters $\lambda$ and $\mu$ are given by (one may apply the Lagrange multiplier technique and optimize the function $\Phi(\mathbf{w})=\mathbf{w}^{H} \mathbf{R}_{2} \mathbf{w}+$ $\beta\left(\mathbf{w}^{H} \mathbf{s}_{A}-1\right)+\rho \mathbf{w}^{H} \boldsymbol{s}_{B}$ with respect to $\mathbf{w}$ where $\beta, \rho$ are arbitrary parameters. As a result, $\partial \Phi / \partial \mathbf{w}=0$ gives us $\mathbf{w}=$ $\left.\lambda \mathbf{R}_{2}^{-1} \mathbf{s}_{A}+\mu \mathbf{R}_{2}^{-1} \mathbf{s}_{B}\right)$

$$
\left(\begin{array}{cc}
\mathbf{s}_{A}^{H} \mathbf{R}_{2}^{-1} \mathbf{s}_{A} & \mathbf{s}_{B}^{H} \mathbf{R}_{2}^{-1} \mathbf{s}_{A} \\
\mathbf{s}_{A}^{H} \mathbf{R}_{2}^{-1} \mathbf{s}_{B} & \mathbf{s}_{B}^{H} \mathbf{R}_{2}^{-1} \mathbf{s}_{B}
\end{array}\right)\left(\begin{array}{l}
\lambda^{*} \\
\mu^{*}
\end{array}\right)=\left(\begin{array}{l}
1 \\
0
\end{array}\right) .
$$

As the search function $T_{s}(n)$ scans through all potential lag values, one is able to identify the points at which a corresponding delayed version of the look direction signal (in this example it is the first source) is encountered as seen in the next section.

$$
\begin{aligned}
& \text { Denoting } \mathbf{R}_{x}=\rho_{1}^{2} \mathbf{s}_{1} \mathbf{s}_{1}^{H}+\mathbf{R}_{1} \text {, we have } \\
& \mathbf{R}_{1}=\rho_{2}^{2} \mathbf{s}_{2} \mathbf{s}_{2}^{H}+\left|\beta_{1,1}\right|^{2} \rho_{1}^{2} \mathbf{s}_{1,1} \mathbf{s}_{1,1}^{H}+\left|\beta_{2,1}\right|^{2} \rho_{2}^{2} \mathbf{s}_{2,1} \mathbf{s}_{2,1}^{H}+\sigma_{n}^{2} \mathbf{I}_{N} .
\end{aligned}
$$

(The case of more than two sources and many number of multipaths does not alter the theory to follow, this is discussed in detail in Appendix A).

3.2. Analysis of the Multipath Finder. Now, for the sake of convenience we represent the $2 N \times 1$ space fast-time weights vector as $\mathbf{w}^{T}=\left(\mathbf{w}_{1}^{T}, \mathbf{w}_{2}^{T}\right)^{T}$, where $N \times 1$ vector $\mathbf{w}_{1}$ refers to the first $N$ components of $\mathbf{w}$ and the rest is represented by $N \times 1$ vector $\mathbf{w}_{2}$. First suppose that the chosen lag $n$ is not equal to any of the values $n_{1, j}(j=1,2, \ldots)$. In this case substituting (3) and $\mathbf{R}_{x}=\rho_{1}^{2} \mathbf{s}_{1} \mathbf{s}_{1}^{H}+\mathbf{R}_{1}$ in $P_{\text {out }}=\mathbf{w}^{H} \mathbf{R}_{2} \mathbf{w}$ we have

$$
P_{\text {out }}=\mathbf{w}_{1}^{H} \mathbf{R}_{1} \mathbf{w}_{1}+\mathbf{w}_{2}^{H} \mathbf{R}_{1} \mathbf{w}_{2}+\rho_{1}^{2} \mathbf{w}_{1}^{H} \mathbf{s}_{1} \mathbf{s}_{1}^{H} \mathbf{w}_{1}+\rho_{1}^{2} \mathbf{w}_{2}^{H} \mathbf{s}_{1} \mathbf{s}_{1}^{H} \mathbf{w}_{2} .
$$

The minimization of power subject to the same constraints: $\mathbf{w}^{H} \mathbf{s}_{A}=1$ and $\mathbf{w}^{H} \boldsymbol{s}_{B}=0$ (i.e., $\mathbf{w}_{1}^{H} \mathbf{s}_{1}=1$ and $\mathbf{w}_{2}^{H} \mathbf{s}_{1}=0$ ) leads to the following solution:

$$
\mathbf{w}_{1}=\frac{\mathbf{R}_{1}^{-1} \mathbf{s}_{1}}{\left(\mathbf{s}_{1}^{H} \mathbf{R}_{1}^{-1} \mathbf{s}_{1}\right)}, \quad \mathbf{w}_{2}=\mathbf{o}_{N \times 1} .
$$

(Note: this procedure cannot be used to find the weights, the earlier described process must be applied to evaluate the space fast-time weights vector).

In this case we have the following expression for the space fast-time processor output power:

$$
\begin{aligned}
P_{\text {out }} & =\mathbf{w}^{H} \mathbf{R}_{2} \mathbf{w} \\
& =\mathbf{w}_{1}^{H} \mathbf{R}_{1} \mathbf{w}_{1}+\rho_{1}^{2} \mathbf{w}_{1}^{H} \mathbf{s}_{1} \mathbf{s}_{1}^{H} \mathbf{w}_{1} \\
& =\left(\mathbf{s}_{1}^{H} \mathbf{R}_{1}^{-1} \mathbf{s}_{1}\right)^{-1}+\rho_{1}^{2} .
\end{aligned}
$$

Substituting this expression in (6) leads to

$$
T_{S}(n)_{n \neq n_{1,1}}=\frac{\left(\mathbf{s}_{1}^{H} \mathbf{R}_{x}^{-1} \mathbf{s}_{1}\right)^{-1}}{\left(\mathbf{s}_{1}^{H} \mathbf{R}_{1}^{-1} \mathbf{s}_{1}\right)^{-1}+\rho_{1}^{2}}-1=0 .
$$

(See Appendix B for a proof of the result $\left(\mathbf{s}_{1}^{H} \mathbf{R}_{x}^{-1} \mathbf{s}_{1}\right)^{-1}=$ $\left.\left(\mathbf{s}_{1}^{H} \mathbf{R}_{1}^{-1} \mathbf{s}_{1}\right)^{-1}+\rho_{1}^{2}\right)$. It was noticed that $\mathbf{w}_{2}=\mathbf{o}_{N \times 1}$ if and only if $\mathbf{Q}=\mathbf{O}_{N \times N}$. As a result we would consider the scaled quantity $T_{s}(n)=\left(P_{\text {out }}-\mathbf{w}_{1}^{H} \mathbf{R}_{1} \mathbf{w}_{1}-\rho_{1}^{2} \mathbf{w}_{1}^{H} \mathbf{s}_{1} \mathbf{s}_{1}^{H} \mathbf{w}_{1}\right) / P_{\text {out }}$, which is a function of $\mathbf{w}_{2}$ only, as a suitable multipath lag finder. Further simplification of this quantity using the look direction constraints, the result in Appendix B, and (10) leads to (6).

The most important fact here is that we do not have to assume the simple case of a mainlobe source and one multipath path to prove that this quantity is zero. The finder spectrum has the following properties, as we look into the direction $\left(\phi_{1}, \theta_{1}\right)$ :

$$
T_{S}(n) \approx \begin{cases}P_{\text {out }}^{-1}\left(\mathbf{s}_{1}^{H} \mathbf{R}_{x}^{-1} \mathbf{s}_{1}\right)^{-1}-1, & n=n_{1, j} \text { for some } j, \\ 0, & n \neq n_{1, j} .\end{cases}
$$


This can be further simplified to obtain the following property (Appendix A):

$$
T_{S}(n)= \begin{cases}N\left|\beta_{1, j}\right|^{2} \mathrm{snr}_{1}, & n=n_{1, j} \text { for some } j, \\ 0, & n \neq n_{1, j} .\end{cases}
$$

This spectrum indicates an infinite processing gain (at least in theory) and is able to detect extremely small power due to multipath off the mainlobe source while suppressing the source (transmitter) itself and any of the unrelated sidelobe arrivals and their multipaths. Furthermore, we can arrive at the following results.

In order to quantify the processing gain of this spectrum one has to replace the zero figure with a quantity which would represent the average output interference level present in the spectrum whenever a lag mismatch occurs. Replacing $\mathbf{Q}^{H}=\mathbf{O}_{N \times N}$ in (3) by an approximate figure (when $n \neq n_{1,1}$ ) would give rise to a small nonzero value. This figure can be shown to be of the order $N / M s n r_{1}$ (written as $O\left(N / M s n r_{1}\right)$ ), where $M$ is the number of samples used in covariance averaging. As a result we can establish processing gain as

$$
\frac{T_{S}(n)_{n=n_{1,1}}}{T_{S}(n)_{n \neq n_{1,1}}} \approx \frac{N\left|\beta_{1, j}\right|^{2} \mathrm{snr}_{1}}{O\left(N / M \operatorname{snr}_{1}\right)} \approx O\left(M\left|\beta_{1, j}\right|^{2} \mathrm{snr}_{1}^{2}\right) .
$$

(See Appendix A for the proof). This equation allows us to establish the following lemma.

Lemma 1. In order to detect a very small multipath power level of the order $1 / N$ (i.e., $\left|\beta_{1 j}\right|^{2} \approx 1 / N$ while satisfying $\operatorname{snr}_{1}>1$ ), with a processing gain of approximately $10 \mathrm{~dB}$ (value at peak point when a match occurs/the average output level when a mismatch occurs), one needs to average around $10 \mathrm{~N}(=M)$ samples at the covariance matrix. However if $\mathrm{snr}_{1}$ is large (i.e., $\gg 1$ ) one can use fewer samples.

For example, if $\operatorname{snr}_{1}=10 \mathrm{~dB}$, then any value of $N(>$ $M)$ can produce $10 \mathrm{~dB}$ processing gain at the spectrum for multipath signals of order $\left|\beta_{1 j}\right|^{2} \approx 1 / N$. In fact simulations generally show much better processing gains as discussed later.

\section{Mutipath Bearing Estimator}

4.1. MPDR Solution. In order to estimate the direction of arrival of the multipath signals, we apply a modified version of the traditionally used Minimum Power Distortionless Response (MPDR) approach [15]. The fundamental assumption we make in this section is that one is able to identify all the associated time lags of the look direction signal (transmitter). The remaining issue we need to resolve here is to estimate the direction of arrival of all the multipaths in the azimuth/elevation plane. Assume as in (4) we have selected the desired delay factor $\left(n_{1,1}\right)$ to form the space-fast time data vector. The $2 N \times 2 N$ signal covariance matrix formed by summing and averaging the outer products $\mathbf{X}_{n_{1,1}}(t) \mathbf{X}_{n_{1,1}}(t)^{H}$ has the following properties. Its signal subspace, which is a subspace of complex
$2 N$ dimensional space (or $\mathbf{C}^{2 N \times 1}$ ), formed by the base vectors $\left(\mathbf{s}_{1}^{T}, \beta_{1,1} \mathbf{s}_{1,1}^{T}\right)^{T},\left(\mathbf{o}_{N \times 1}^{T}, \mathbf{s}_{1}^{T}\right)^{T},\left(\mathbf{s}_{1,1}^{T}, \mathbf{o}_{N \times 1}^{T}\right)^{T},\left(\mathbf{s}_{2}^{T}, \mathbf{o}_{N \times 1}^{T}\right)^{T}$, $\left(\mathbf{o}_{N \times 1}^{T}, \mathbf{s}_{2}^{T}\right)^{T},\left(\mathbf{s}_{2,1}^{T}, \mathbf{o}_{N \times 1}^{T}\right)^{T}$, and $\left(\mathbf{o}_{N \times 1}^{T}, \mathbf{s}_{2,1}^{T}\right)^{T}$ (more base vectors may exist due to more sources and associated multipaths, this will not alter the argument to follow). For any given arbitrary $\mathbf{s}(\phi, \theta)$ consider the space fast-time steering vector constructed by $\mathbf{S}(\phi, \theta, \beta)^{T}=\left(\mathbf{s}_{1}\left(\phi_{1}, \theta_{1}\right)^{T}, \beta \mathbf{s}(\phi, \theta)^{T}\right)^{T}$, where $\beta$ is a variable. As $\phi, \theta, \beta$ vary over all possible values, the two steering vectors $\mathbf{S}\left(\phi, \theta, \beta_{1}\right)^{T}=\left(\mathbf{s}_{1}\left(\phi_{1}, \theta_{1}\right)^{T}, \beta_{1} \mathbf{s}(\phi, \theta)^{T}\right)^{T}$ and $\mathbf{S}\left(\phi, \theta, \beta_{2}\right)^{T}=\left(\mathbf{s}_{1}\left(\phi_{1}, \theta_{1}\right)^{T}, \beta_{2} \mathbf{s}(\phi, \theta)^{T}\right)^{T}$ are linearly independent whenever $\beta_{1} \neq \beta_{2}$.

Now if we minimize $\mathbf{W}^{H} \mathbf{R}_{2} \mathbf{W}$ subject to $\mathbf{W}^{H} \mathbf{S}(\phi, \theta, \beta)=$ 1 by choosing an arbitrary value for $\beta$ (where $\beta \neq \beta_{1, j}, j=$ $1,2, \ldots)$, the natural tendency is to provide a solution $\mathbf{W}$ that is almost orthogonal to all the base vectors (which includes $\left.\mathbf{S}\left(\phi_{1,1}, \theta_{1,1}, \beta_{1,1}\right)=\left(\mathbf{s}_{1}^{T}, \beta_{1,1} \mathbf{s}_{1,1}^{T}\right)^{T}\right)$ in signal subspace mentioned earlier. The reason for this is that the look direction vector $\mathbf{S}(\phi, \theta, \beta)$ does not represent any vector in the signal subspace. However, if we choose $\mathbf{S}\left(\phi_{1,1}, \theta_{1,1}, \beta_{1,1}\right)=$ $\left(\mathbf{s}_{1}^{T}, \beta_{1,1} \mathbf{s}_{1,1}^{T}\right)^{T}$ (yet unknown) as the look direction vector, we would receive energy corresponding to this vector while minimizing the energy due to all other direction of arrivals. Therefore, if we find a set of values for $\phi, \theta, \beta$ in order to optimize $\mathbf{W}^{H} \mathbf{R}_{2} \mathbf{W}$, then the only available solution is $\phi_{1,1}, \theta_{1,1}, \beta_{1,1}$.

A suitable procedure to achieve this result is to first optimise $\mathbf{W}^{H} \mathbf{R}_{2} \mathbf{W}$ for a fixed $\beta$ and then further optimize the output with respect to $\beta$, this way, one is expected to reach a maxima for the quantity $\mathbf{W}^{H} \mathbf{R}_{2} \mathbf{W}$ at the correct value of $\phi, \theta, \beta$ which represent $\left(\mathbf{s}_{1}^{T}, \beta_{1,1} \mathbf{s}_{1,1}^{T}\right)^{T}$ while minimizing the energy content in the output due to all other signals in the signal subspace.

Now consider

$$
\Phi(\phi, \theta, \beta)=\mathbf{W}^{H} \mathbf{R}_{2} \mathbf{W}+\lambda\left(\mathbf{W}^{H} \mathbf{S}(\phi, \theta, \beta)-1\right) .
$$

By applying the Lagrange Multiplier technique we have

$$
\mathbf{W}=-\lambda \mathbf{R}_{2}^{-1} \mathbf{S}(\phi, \theta, \beta),
$$

where $\lambda$ is given by $\mathbf{W}^{H} \boldsymbol{S}(\phi, \theta, \beta)=1$. As a result we have

$$
\Phi(\phi, \theta, \beta)^{-1}=\mathbf{S}(\phi, \theta, \beta)^{H} \mathbf{R}_{2}^{-1} \mathbf{S}(\phi, \theta, \beta) .
$$

Further differentiation of this quantity is carried out by rewriting (18) in the following form:

$$
\begin{aligned}
\Phi(\phi, \theta, \beta)^{-1} \\
=\left[\left(\begin{array}{c}
\mathbf{s}_{1} \\
\mathbf{o}_{N \times 1}
\end{array}\right)+\beta\left(\begin{array}{c}
\mathbf{o}_{N \times 1} \\
\mathbf{s}
\end{array}\right)\right]^{H} \mathbf{R}_{2}^{-1}\left[\left(\begin{array}{c}
\mathbf{s}_{1} \\
\mathbf{o}_{N \times 1}
\end{array}\right)+\beta\left(\begin{array}{c}
\mathbf{o}_{N \times 1} \\
\mathbf{s}
\end{array}\right)\right] \\
=\left(\widehat{\boldsymbol{S}}_{1}+\beta \widehat{\mathbf{S}}\right)^{H} \mathbf{R}_{2}^{-1}\left(\widehat{\boldsymbol{S}}_{1}+\beta \widehat{\boldsymbol{S}}\right) \\
\Phi(\phi, \theta, \beta)^{-1} \\
=\widehat{\mathbf{S}}_{1}^{H} \mathbf{R}_{2}^{-1} \widehat{\mathbf{S}}_{1}+\beta^{*} \widehat{\mathbf{S}}_{H} \mathbf{R}_{2}^{-1} \widehat{\mathbf{S}}_{1}+\beta \widehat{\mathbf{S}}_{1}^{H} \mathbf{R}_{2}^{-1} \widehat{\mathbf{S}} \\
+|\beta|^{2} \widehat{\mathbf{S}}_{H} \mathbf{R}_{2}^{-1} \widehat{\mathbf{S}}
\end{aligned}
$$

where, $\widehat{\mathbf{S}}_{1}=\left(\mathbf{s}_{1}, o_{N \times 1}^{T}\right)^{T}$ and $\widehat{\mathbf{S}}=\left(\mathbf{o}_{N \times 1}^{T}, \mathbf{s}^{T}\right)^{T}$. 
Now $\partial \Phi^{-1} / \partial \beta^{*}=0$ gives

$$
\beta=-\frac{\left(\widehat{\mathbf{S}}^{H} \mathbf{R}_{2}^{-1} \widehat{\mathbf{S}}_{1}\right)}{\widehat{\mathbf{S}}^{H} \mathbf{R}_{2}^{-1} \widehat{\mathbf{S}}}
$$

For every given value of the pair $(\phi, \theta)$ we can estimate $\beta$ using (20) and plot $\Phi$ in the $(\phi, \theta)$ plane in order to obtain the peak point which occurs at $\left(\phi_{1,1}, \theta_{1,1}, \beta_{1,1}\right)$ point only. This procedure is carried out for every multipath detected using the lag finder.

4.2. High-Resolution Approach. Suppose $\mathbf{e}_{1}, \mathbf{e}_{2}, \ldots, \mathbf{e}_{M}$ represent the signal subspace eigen vector of $\mathbf{R}_{2}$. Here the value of $M$ is selected using the usual rules used in the MUSIC technique $[16,17]$. Assuming that this parameter is found using the eigen analysis of $\mathbf{R}_{2}$ we apply the following argument. The steering vector $\mathbf{S}(\phi, \theta, \beta)^{T}=\left(\mathbf{s}_{1}\left(\phi_{1}, \theta_{1}\right)^{T}, \beta \mathbf{s}(\phi, \theta)^{T}\right)^{T}$ corresponding to any signal in the signal subspace is a linear combination of the eigen vectors $\mathbf{e}_{1}, \mathbf{e}_{2}, \ldots, \mathbf{e}_{M}$. We may write this as

$$
\mathbf{S}(\phi, \theta, \beta)^{T}=\mathbf{E A},
$$

where $\mathbf{E}=\mathbf{e}_{1}, \mathbf{e}_{2}, \ldots, \mathbf{e}_{M}, \mathbf{A}=\left(a_{1}, a_{2}, \ldots, a_{M}\right)^{T}$ and $a_{1}, a_{2}, \ldots, a_{M}$ represent a set of unknown parameters. This linear system is satisfied for some $\mathbf{A}$, only if the correct values of $\beta$ and $(\phi, \theta)$ are encountered, namely, $\beta=\beta_{1,1}$ and $(\phi, \theta)=$ $\left(\phi_{1,1}, \theta_{1,1}\right)$. Any other value for these parameters would not represent a steering value that corresponds to a signal that exists in the signal subspace. Therefore a suitable spectrum to detect these values would be

$$
\begin{aligned}
F(\phi, \theta, \beta) & =\|\mathbf{V S}(\phi, \theta, \beta)\|^{2} \\
& =\mathbf{S}(\phi, \theta, \beta)^{H} \mathbf{V}^{H} \mathbf{V S}(\phi, \theta, \beta),
\end{aligned}
$$

where

$$
\mathbf{V}=\left[\mathbf{I}_{2 N \times 2 N}-\mathbf{E}\left(\mathbf{E}^{H} \mathbf{E}\right)^{-1} \mathbf{E}^{H}\right]
$$

(known as the projection operator).

Further simplification of (22) leads to

$$
\begin{aligned}
F= & \mathbf{S}_{1}^{H} \mathbf{V}^{H} \mathbf{V} \widehat{S}_{1}+\beta^{*} \widehat{\boldsymbol{S}}_{H} \mathbf{V}^{H} \mathbf{V} \hat{\boldsymbol{S}}_{1} \\
& +\beta \hat{\boldsymbol{S}}_{1}^{H} \mathbf{V}^{H} \mathbf{V S}+\beta \beta^{*} \hat{\boldsymbol{S}}_{H} \mathbf{V}^{H} \mathbf{V} \widehat{\mathbf{S}},
\end{aligned}
$$

the best solution for $\beta$ is obtained by (for every given $\phi, \theta$ ). $\partial F / \partial \beta^{*}=0$, which leads to the solution

$$
\beta=-\frac{\left(\hat{\boldsymbol{S}}^{H} \mathbf{V}^{H} \mathbf{V} \hat{\boldsymbol{S}}_{1}\right)}{\hat{\boldsymbol{S}}^{H} \mathbf{V}^{H} \mathbf{V} \hat{\mathbf{S}}} .
$$

\section{Target Location}

The path delay and the direction of arrival of each multipath can uniquely identify each target location (distance) as follows. As illustrated in Figure 1, the distance between the transmitter and the receiver is assumed to be a known value $d$, the distance to the target from the transmitter is $s_{2}$ (unknown) and the distance from the receiver to the target is $s_{1}$ (unknown), the multipath delay is a known value $\tau$ (estimated using lag finder). Once the bearing estimator has estimated the direction of the arrival of the multipath with lag $\tau$, it is equivalent to the knowledge of the angle $\gamma$ (whenever the transmitter direction is preciously known). Thus we have

$$
s_{1}+s_{2}=d+c \tau,
$$

where $c$ is the speed of light.

Furthermore we have

$$
\left(d-s_{1} \cos \gamma\right)^{2}+\left(s_{1} \sin \gamma\right)^{2}=s_{2}^{2},
$$

therefore we have $s_{2}^{2}-s_{1}^{2}=d^{2}-2 d s_{1} \cos \gamma=\left(s_{2}-s_{1}\right)\left(s_{2}+s_{1}\right)$. Now substituting (26) in the above expression, we have

$$
(d+c \tau)^{2}-2(d+c \tau) s_{1}=d^{2}-2 d s_{1} \cos \gamma,
$$

which leads to the target distance

$$
s_{1}=\frac{c \tau(2 d+c \tau)}{2[c \tau+d(1-\cos \gamma)]} .
$$

\section{Simulation Results}

It should be noted that in this study the primary assumption is that the target and source transmitter are both in the line-of-sight to achieve a perfect correlation of the direct signal with the reflection off the target. Once we identify all available lag values corresponding to all available multipaths of the look direction signal, the multipath bearing estimator estimates the associated direction of arrival for all multipaths which may include reflections off the ground and other stationary points. At this stage most multipaths may be ignored as ground reflections if the associated elevation angle of the multipath is negative. Other reflection points may be tracked over time to validate if they are moving targets, and hence the associated velocities can be estimated.

In the example simulated, we have an array of $16 \times 19$ elements and considered the case with 4 target returns (4 multipaths of the transmitter correspoinding to 4 bistatic radar responses which is on the broadside $\left(\phi_{1}, \theta_{1}\right)=$ $\left.\left(0^{0}, 0^{0}\right)\right)$. The directions of arrivals pairs $\left(\left(\phi_{1, j}, \theta_{1, j}\right), j=\right.$ $1,2,3,4)$ for the multipaths are $\left(10^{0},-10^{0}\right),\left(20^{0},-20^{0}\right)$, $\left(25^{0},-25^{0}\right),\left(30^{0},-30^{0}\right)$. The simulated path delays are 30 , 50,82 , and 84 , respectively. The squares of the reflective coefficients $\left(\left|\beta_{1, j}\right|^{2}, j=1,2,3,4\right)$ are $1 / 20,1 / 30,1 / 30$, and $1 / 30$, respectively. A jammer is present in the direction $\left(\phi_{2}, \theta_{2}\right)=\left(40^{0}, 0^{0}\right)$ with a jammer to noise ratio of $10 \mathrm{~dB}$ and a single multipath of the jammer with $\left(\phi_{2,1}, \theta_{2,1}\right)=$ $\left(5^{0}, 0^{0}\right)$ and $\left|\beta_{2,1}\right|^{2}=1 / 10$. We have considered the two cases where the transmitter power to noise ratio $\mathrm{snr}_{1}=$ $7 \mathrm{~dB}$, and $\operatorname{snr}_{1}=-10 \mathrm{~dB}$. The Lag finder spectrum is 


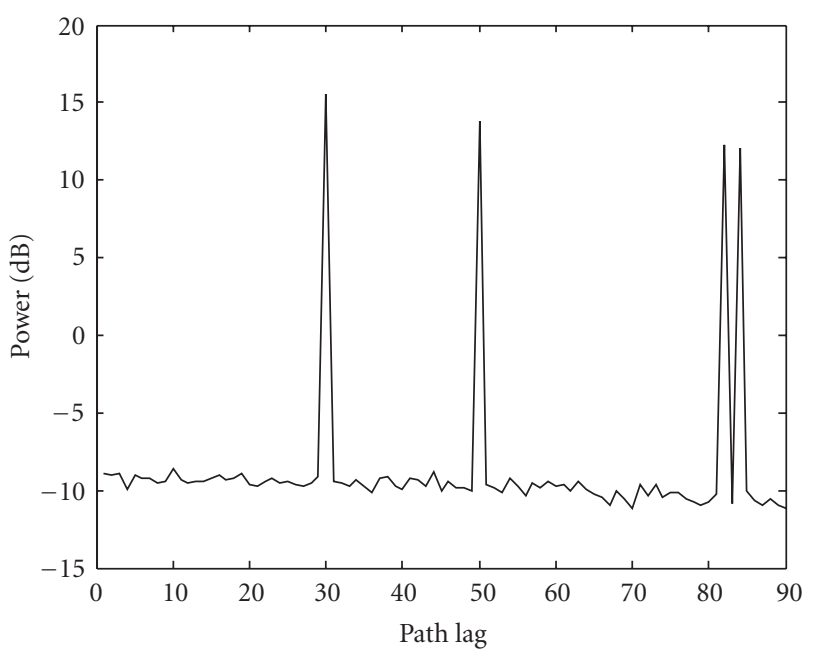

(a)

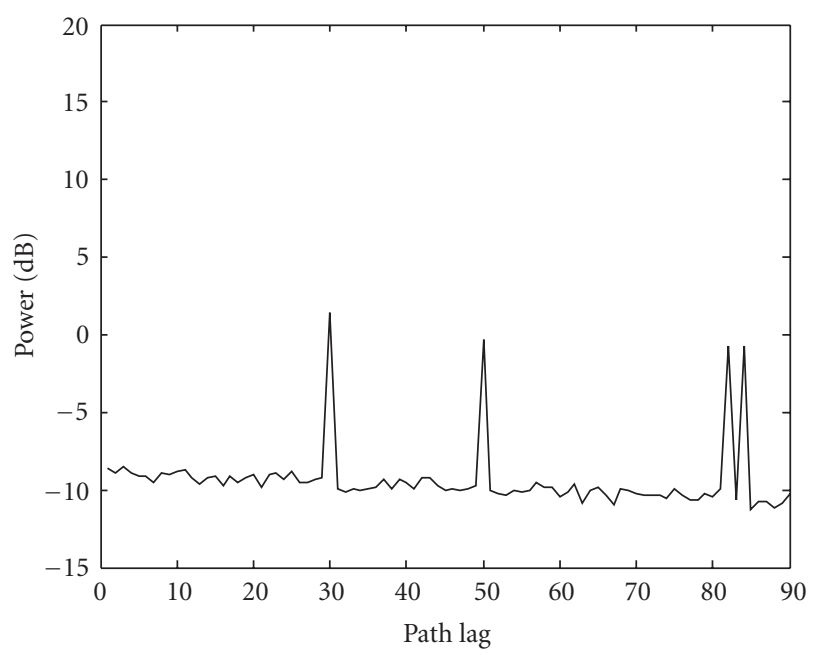

(b)

Figure 2: (a) Multipath lag finder spectrum when the look direction is the broadside with $\mathrm{snr}_{1}=7 \mathrm{~dB}$. (b) Path lag finder spectrum when the look direction is the broadside with $\mathrm{snr}_{1}=-10 \mathrm{~dB}$.

shown in Figures 2(a) and 2(b), respectively, of the two cases. This demonstrates the fact that the theory works very well for the case $\operatorname{snr}_{1} \leq 1$. But this case was not analyzed due to the mathematical complexity involved. It should be noted that for the case $\mathrm{snr}_{1}=7 \mathrm{~dB}$, we have the received target reflectivity power to noise power ratios of (i.e., $\mathrm{snr}_{j}$. $\left.\left|\beta_{1 j}\right|^{2}, j=1,2,3,4\right)-6 \mathrm{~dB},-8 \mathrm{~dB},-8 \mathrm{~dB}$, and $-8 \mathrm{~dB}$, respectively.

Once the Lag finder spectrum identifies the lag values available, one has to produce the angle of arrival estimate spectrum as shown in Figure 3(a) or 3(b) using the MPDR or high-resolution solution for each lag value. This spectrum accurately estimates the azimuth and elevation values as well as the reflective coefficient for each multipath. Figure 4 displays the results of the 4 multipaths we have estimated using this procedure (horizontal and vertical cuts across the peak points of the azimuth/elevation plots for all of

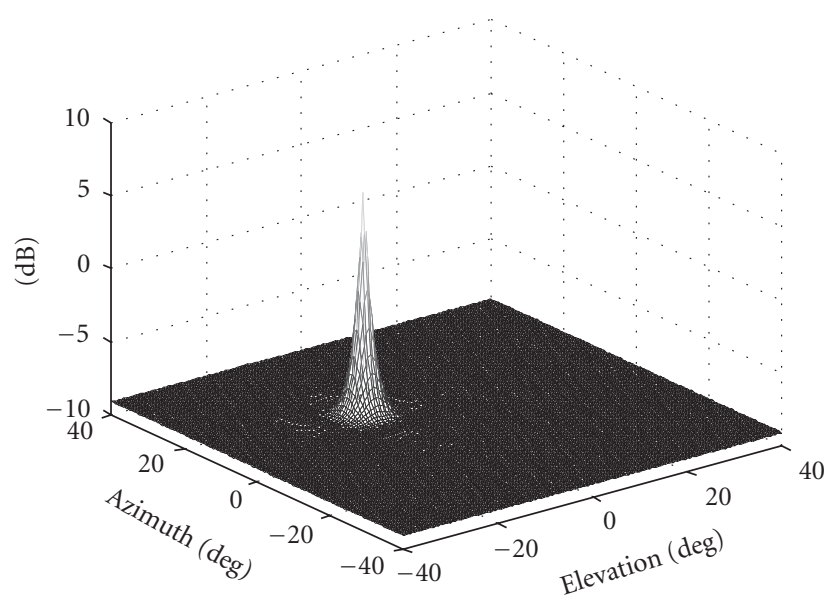

(a)

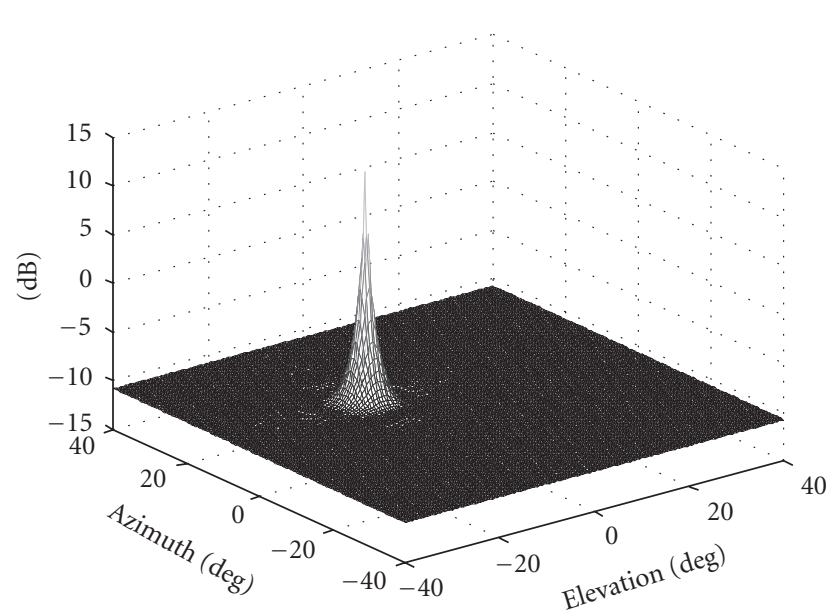

(b)

FIGURE 3: (a) MPDR solution for the lag $=30\left(\operatorname{snr}_{1}=7 \mathrm{~dB}\right)$. (b) high-resolution (HR) solution for the lag $=30\left(\operatorname{snr}_{1}=7 \mathrm{~dB}\right)$.

lag values). This procedure can identify all target directions of arrivals. Figure 5 illustrates the estimated value and the exact values of a montecarlo simulation run where $\beta_{1,1}$ and $\beta_{1,2}$ assume various values (one decreases while the other increases, keeping $3 \mathrm{rd}$ and 4 th multipath reflectivity coefficients (squared) constant values of 1/30 each). In Figure $5,+$ or $*$ denotes the average estimate for the parameter, while straight lines represent its exact value. When the multipath contributions are of extended nature, namely, ground scatter, one would expect a cluster of peak points in the TSI domain extending over several lag values (Figure 2). In theory, as long as we consider the middle value (lag) as the solution to form the correct space fast-time processor, we can implement multipath bearing estimator and subsequently employ the triangulation technique to identify the origin (reflection point).

As to the computation cost, the usual spatial beamformer generally requires $O\left(N^{3}\right)$ operations to perform the matrix inversion for an $N$ element array where the size of the matrix is $N \times N$. However, for the same array, the space-time 


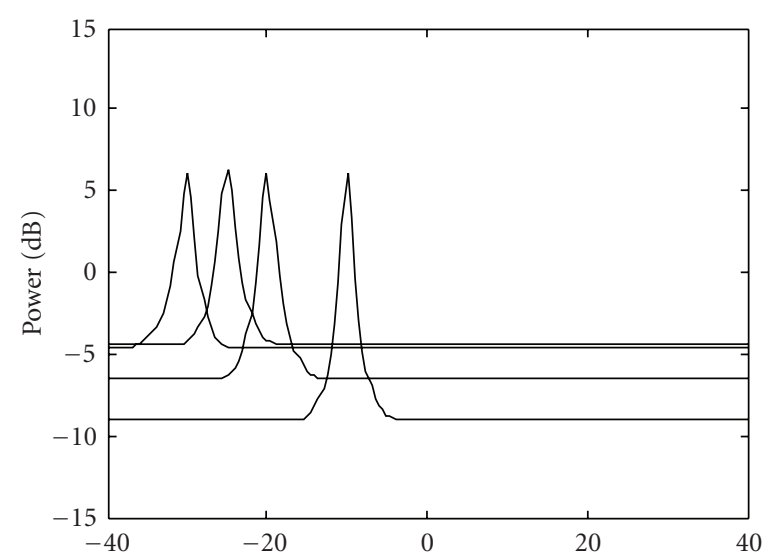

(a) Elevation (deg)-MPDR solution

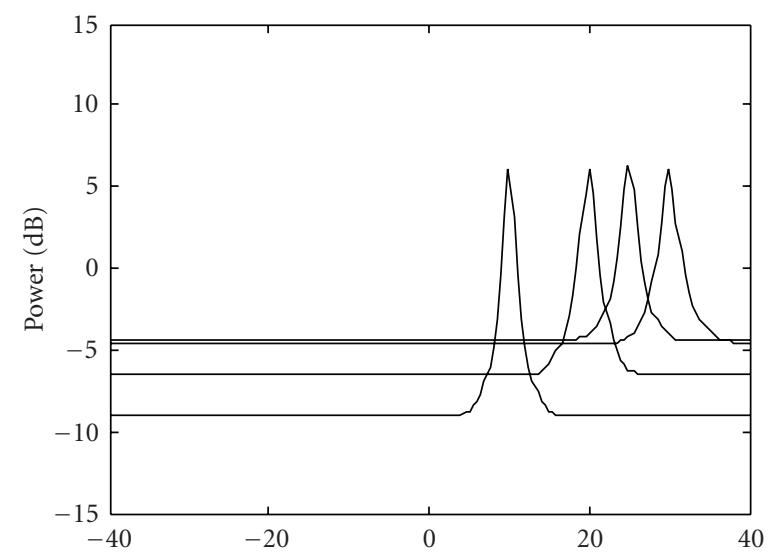

(c) Azimuth (deg)-MPDR solution

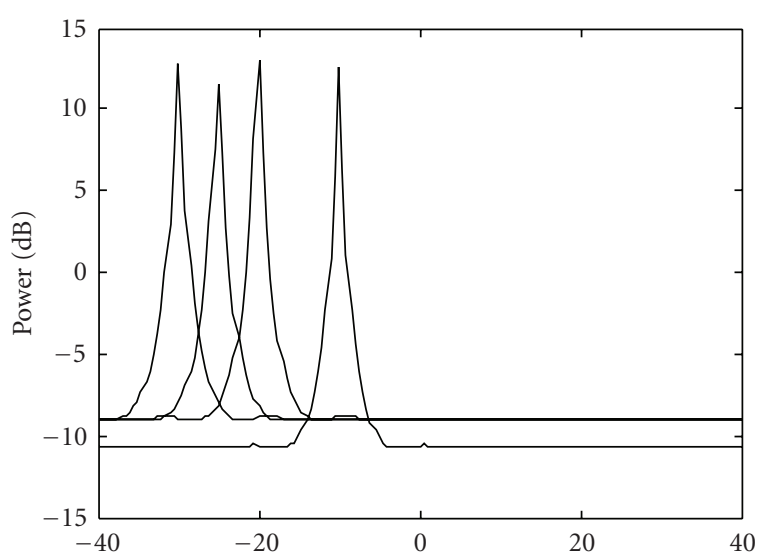

(b) Elevation (deg)-HR solution

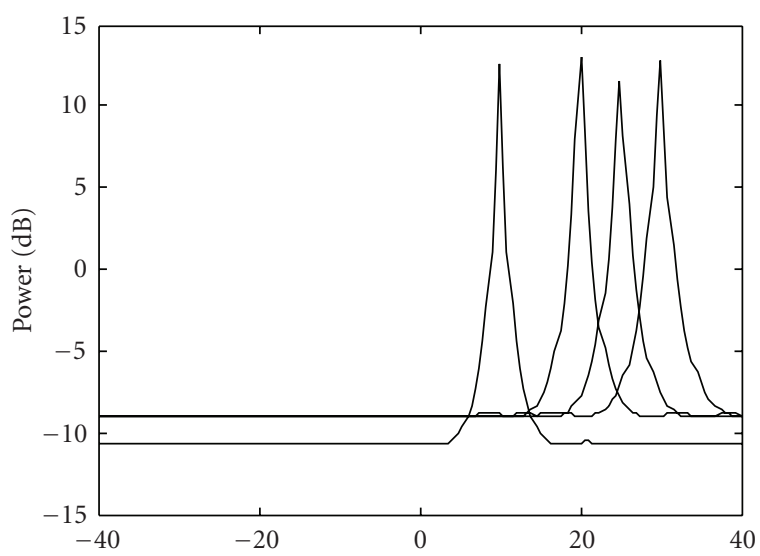

(d) Azimuth (deg)-HR solution

FIGURE 4: Bearing estimation for all four multipaths using all four lag estimates. These figures display the cuts across the peak values of the elevation/azimuth spectrum of the type displayed in Figure 3.

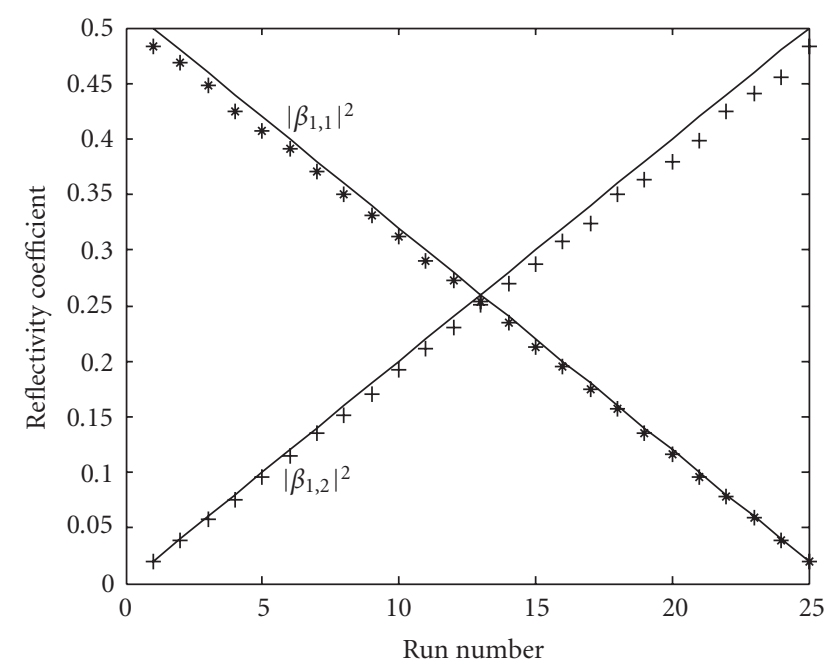

Figure 5: The estimated value of the reflectivity parameters $\beta_{1,1}$ and $\beta_{1,2}$ with $\left|\beta_{1,3}\right|^{2}=\left|\beta_{1,4}\right|^{2}=1 / 30$. Straight lines represent the simulated values, and $*$ or + represents the estimations.

beamformer inverts a larger matrix of size $2 N \times 2 N$. This procedure increases the computation load by a factor 8 .

\section{Concluding Remarks}

We have simulated the existing lag finding algorithm (or better known as TSI finder) to estimate all the delays corresponding to multipath arrivals due to bistatic radar responses present in the received signal where the received signal (main beam signal) is generally a known transmitter. Once all its multipaths are located in the lag domain, a new postprocessor algorithm was developed for multipath direction finding. We used two approaches to evaluate the target bearings of all the reflected paths due to a known signal of interest. Simulation shows the high-resolutionbased approach always provides better signal processing gain at a higher computational cost (around 100\% more). Furthermore, the simulation study has shown that when the time delay of the reflected path is not an integer multiple of the sample size (range sample size), it did not reduce the spectrum's performance more than $3 \mathrm{~dB}$ in the lag finding spectrum. The proposed algorithm is robust and flexible and may lend itself to many applications as discussed in the introduction. The use of the transmitter of opportunity is possible only if the transmitter's bearing and the position are known. 


\section{Appendices}

\section{A.}

The output power at the processor $P_{\text {out }}\left(\right.$ for $\left.n=n_{1,1}\right)$ given by (using (5) and substituting $\mathbf{R}_{x}=\rho_{1}^{2} \mathbf{s}_{1} \mathbf{s}_{1}^{H}+\mathbf{R}_{1}$ )

$$
\begin{aligned}
P_{\text {out }}= & \mathbf{w}^{H} \mathbf{R}_{2} \mathbf{w} \\
= & \mathbf{w}_{1}^{H} \mathbf{R}_{1} \mathbf{w}_{1}+\mathbf{w}_{2}^{H} \mathbf{R}_{1} \mathbf{w}_{2} \\
& +\rho_{1}^{2} \mathbf{w}_{1}^{H} \mathbf{s}_{1} \mathbf{s}_{1}^{H} \mathbf{w}_{1}+\rho_{1}^{2} \mathbf{w}_{2}^{H} \mathbf{s}_{1} \mathbf{s}_{1}^{H} \mathbf{w}_{2} \\
& +\rho_{1}^{2} \beta_{1,1}^{*} \mathbf{w}_{1}^{H} \mathbf{s}_{1} \mathbf{s}_{1,1}^{H} \mathbf{w}_{2}+\rho_{1}^{2} \beta_{1,1} \mathbf{w}_{2}^{H} \mathbf{s}_{1,1} \mathbf{s}_{1}^{H} \mathbf{w}_{1} .
\end{aligned}
$$

When the constraints $\mathbf{w}_{1}^{H} \mathbf{s}_{1}=1.0$ and $\mathbf{w}_{2}^{H} \mathbf{s}_{1}=0$ are imposed, we have

$$
\begin{aligned}
P_{\text {out }}= & \mathbf{w}^{H} \mathbf{R}_{2} \mathbf{w} \\
= & \mathbf{w}_{1}^{H} \mathbf{R}_{1} \mathbf{w}_{1}+\mathbf{w}_{2}^{H} \mathbf{R}_{1} \mathbf{w}_{2}+\rho_{1}^{2} \\
& +\rho_{1}^{2}\left(\beta_{1,1}^{*} \mathbf{s}_{1,1}^{H} \mathbf{w}_{2}+\beta_{1,1} \mathbf{w}_{2}^{H} \mathbf{s}_{1,1}\right) .
\end{aligned}
$$

The original power minimization problem can now be broken into two independent minimization problems as follows.

(1) Minimize $\mathbf{w}_{1}^{H} \mathbf{R}_{1} \mathbf{w}_{1}$ subject to the constraint $\mathbf{w}_{1}^{H} \mathbf{s}_{1}=$ 1.

(2) Minimize $\mathbf{w}_{2}^{H} \mathbf{R}_{1} \mathbf{w}_{2}+\rho_{1}^{2}+\rho_{1}^{2}\left(\beta_{1,1}^{*} \mathbf{s}_{1,1}^{H} \mathbf{w}_{2}+\beta_{1,1} \mathbf{w}_{2}^{H} \mathbf{s}_{1,1}\right)$ subject to $\mathbf{w}_{2}^{H} \mathbf{s}_{1}=0$.

The solution can be expressed as

$$
\begin{gathered}
\mathbf{w}_{1}=\frac{\mathbf{R}_{1}^{-1} \mathbf{s}_{1}}{\left(\mathbf{s}_{1}^{H} \mathbf{R}_{1}^{-1} \mathbf{s}_{1}\right)}, \\
\mathbf{w}_{2}=-\beta_{1,1} \rho_{1}^{2} \mathbf{R}_{1}^{-1} \mathbf{s}_{1,1}+\beta_{1,1} \rho_{1}^{2}\left(\frac{\mathbf{s}_{1}^{H} \mathbf{R}_{1}^{-1} \mathbf{s}_{1,1}}{\mathbf{s}_{1}^{H} \mathbf{R}_{1}^{-1} \mathbf{s}_{1}}\right) \mathbf{R}_{1}^{-1} \mathbf{s}_{1} .
\end{gathered}
$$

The above representation of the solution cannot be used to compute the space-time weights vector $\mathbf{w}$ due to the fact that the quantities involved are not measurable. Instead the result in (7) is implemented to evaluate $\mathbf{w}$ as described earlier in Section 3.

Substituting $\mathbf{R}_{1}=\rho_{2}^{2} \mathbf{s}_{2} \mathbf{s}_{2}^{H}+\rho_{1}^{2}\left|\beta_{1,1}\right|^{2} \mathbf{s}_{1,1} \mathbf{s}_{1,1}^{H}+$ $\rho_{2}^{2}\left|\beta_{2,1}\right|^{2} \mathbf{s}_{2,1} \mathbf{s}_{2,1}^{H}+\sigma_{n}^{2} \mathbf{I}_{N}$ into (A.2) and noting that $\rho_{1}^{2}\left|\beta_{1,1}\right|^{2} \mathbf{w}_{2}^{H} \mathbf{s}_{1,1} \mathbf{s}_{1,1}^{H} \mathbf{w}_{2}+\rho_{1}^{2}+\rho_{1}^{2}\left(\beta_{1,1}^{*} \mathbf{s}_{1,1}^{H} \mathbf{w}_{2}+\beta_{1,1} \mathbf{w}_{2}^{H} \mathbf{s}_{1,1}\right)=$ $\rho_{1}^{2}\left|1+\beta_{1,1} \mathbf{w}_{2}^{H} \mathbf{s}_{1,1}\right|^{2}$, we have the following expression for the output power:

$$
\begin{aligned}
P_{\text {out }}= & \rho_{1}^{2}\left|\beta_{1,1}\right|^{2}\left|\mathbf{w}_{1}^{H} \mathbf{s}_{1,1}\right|^{2}+\rho_{1}^{2}\left|1+\beta_{1,1} \mathbf{w}_{2}^{H} \mathbf{s}_{1,1}\right|^{2} \\
& +\mathbf{w}_{1}^{H} \mathbf{R}_{0} \mathbf{w}_{1}+\mathbf{w}_{2}^{H} \mathbf{R}_{0} \mathbf{w}_{2}+\sigma_{n}^{2}\left(\mathbf{w}_{1}^{H} \mathbf{w}_{1}+\mathbf{w}_{2}^{H} \mathbf{w}_{2}\right),
\end{aligned}
$$

where $\mathbf{R}_{0}=\rho_{2}^{2} \mathbf{s}_{2} \mathbf{s}_{2}^{H}+\left|\beta_{2}\right|^{2} \rho_{2}^{2} \mathbf{s}_{2,1} \mathbf{s}_{2,1}^{H}$ is the output energy due to any second source and associated multipaths present at the input. It should be noted that this component of the output also contains any output energy due to any second (unmatched) multipath of the look direction source (e.g., $\left|\beta_{1,2}\right|^{2} \mathbf{s}_{1,2} \mathbf{s}_{1,2}^{H}$ terms). The most general form would be

$$
\begin{aligned}
\mathbf{R}_{0}= & \sum_{j=2}^{a_{1}} \rho_{1}^{2}\left|\beta_{1, j}\right|^{2} \mathbf{s}_{1, j} \mathbf{s}_{1, j}^{H}+\sum_{k=2}^{q} \rho_{k}^{2} \mathbf{s}_{k} \mathbf{s}_{k}^{H} \\
& +\sum_{k=2}^{q} \sum_{j=1}^{a_{k}} \rho_{k}^{2}\left|\beta_{k, j}\right|^{2} \mathbf{s}_{k, j} \mathbf{s}_{k, j}^{H},
\end{aligned}
$$

where $q$ is the number of sources and $a_{k}$ is the number of TSI paths available for the $k$ th source. The expression for $P_{\text {out }}$ in (A.5) clearly indicates that the best $\mathbf{w}_{1}$ that (which has a total degrees of freedom $N$ ) would minimize $P_{\text {out }}$ is very likely to be orthogonal to $\mathbf{s}_{1,1}$, that is, $\left|\mathbf{w}^{H} \mathbf{s}_{1,1}\right| \approx 0$ and furthermore it would be attempting to satisfy $\left|1+\beta_{1,1} \mathbf{w}_{2}^{H} \mathbf{s}_{1,1}\right|^{2} \approx 0$ while being orthogonal to all other signals present in $\mathbf{R}_{0}$.

Note that

$$
\begin{aligned}
\mathbf{w}_{1}^{H} \mathbf{R}_{0} \mathbf{w}_{1}= & \sum_{j=2}^{a_{1}} \rho_{1}^{2}\left|\beta_{1, j}\right|^{2}\left|\mathbf{w}_{1}^{H} \mathbf{s}_{1, j}\right|^{2}+\sum_{k=2}^{q} \rho_{k}^{2}\left|\mathbf{w}_{1}^{H} \mathbf{s}_{k}\right|^{2} \\
& +\sum_{k=2}^{q} \sum_{j=1}^{a_{k}} \rho_{k}^{2}\left|\beta_{k, j}\right|^{2}\left|\mathbf{w}_{1}^{H} \mathbf{s}_{k, j}\right|^{2}
\end{aligned}
$$

and a similar expression holds for $\left(\mathbf{w}_{2}^{H} \mathbf{R} \mathbf{w}_{2}\right)$.

Any remaining degrees of freedom would be used to minimize the contribution due to the white noise component. In order to investigate the properties of the solution for $\mathbf{w}$ let us assume that we have only a look direction signal and its mutipath, in which case we have $\mathbf{R}_{0}=\mathbf{O}_{N \times N}$ and

$$
\begin{aligned}
P_{\text {out }}= & \rho_{1}^{2}\left|\beta_{1,1}\right|^{2}\left|\mathbf{w}_{1}^{H} \mathbf{s}_{1,1}\right|^{2}+\rho_{1}^{2}\left|1+\beta_{1,1} \mathbf{w}_{2}^{H} \mathbf{s}_{1,1}\right|^{2} \\
& +\sigma_{n}^{2}\left(\mathbf{w}_{1}^{H} \mathbf{w}_{1}+\mathbf{w}_{2}^{H} \mathbf{w}_{2}\right) .
\end{aligned}
$$

In this case $\mathbf{R}_{1}=\left|\beta_{1,1}\right|^{2} \rho_{1}^{2} \mathbf{s}_{1,1} \mathbf{s}_{1,1}^{H}+\sigma_{n}^{2} \mathbf{I}_{N}$ and the inverse of which is given by

$$
\mathbf{R}_{1}^{-1}=\frac{1}{\sigma_{n}^{2}}\left[\mathbf{I}_{N}-\frac{\left(\rho_{1}^{2}\left|\beta_{1,1}\right|^{2} \mathbf{s}_{1,1} \mathbf{s}_{1,1}^{H}\right)}{\left(\sigma_{n}^{2}+N\left|\beta_{1,1}\right|^{2} \rho_{1}^{2}\right)}\right] .
$$

As a result we have

$$
\begin{aligned}
& \mathbf{R}_{1}^{-1} \mathbf{s}_{1}= \frac{1}{\sigma_{n}^{2}}\left[\mathbf{s}_{1}-\frac{\left(\rho_{1}^{2}\left|\beta_{1,1}\right|^{2} \mathbf{s}_{1,1} \mathbf{s}_{1,1}^{H} \mathbf{s}_{1}\right)}{\left(\sigma_{n}^{2}+N\left|\beta_{1,1}\right|^{2} \rho_{1}^{2}\right)}\right], \\
& \mathbf{R}_{1}^{-1} \mathbf{s}_{1,1}=\frac{\mathbf{s}_{1,1}}{\left(\sigma_{n}^{2}+N\left|\beta_{1,1}\right|^{2} \rho_{1}^{2}\right)},
\end{aligned}
$$




$$
\begin{gathered}
\mathbf{s}_{1,1}^{H} \mathbf{R}_{1}^{-1} \mathbf{s}_{1,1}=\frac{N}{\left(\sigma_{n}^{2}+N\left|\beta_{1,1}\right|^{2} \rho_{1}^{2}\right)}, \\
\mathbf{s}_{1,1}^{H} \mathbf{R}_{1}^{-1} \mathbf{s}_{1}=\frac{\mathbf{s}_{1,1}^{H} \mathbf{s}_{1}}{\left(\sigma_{n}^{2}+N\left|\beta_{1,1}\right|^{2} \rho_{1}^{2}\right)} .
\end{gathered}
$$

Furthermore we adopt the notation $\mathrm{snr}_{1}=\mathrm{snr}$ for the look direction source to noise power and (for $N\left|\beta_{1,1}\right|^{2} \mathrm{snr} \gg 1$ )

$$
\begin{aligned}
\mathbf{s}_{1}^{H} \mathbf{R}_{1}^{-1} \mathbf{s}_{1} & =\frac{1}{\sigma_{n}^{2}}\left[N-\frac{\left(\rho_{1}^{2}\left|\beta_{1,1}\right|^{2}\left|\mathbf{s}_{1}^{H} \mathbf{s}_{1,1}\right|^{2}\right)}{\left(\sigma_{n}^{2}+N\left|\beta_{1,1}\right|^{2} \rho_{1}^{2}\right)}\right] \\
& =\frac{N}{\sigma_{n}^{2}}\left[1-\frac{\left|\mathbf{s}_{1}^{H} \mathbf{s}_{1,1}\right|^{2}\left|\beta_{1,1}\right|^{2} \mathrm{snr}}{N\left(1+N\left|\beta_{1,1}\right|^{2} \mathrm{snr}\right)}\right] \\
& \approx \frac{N}{\sigma_{n}^{2}}\left(1-\frac{\left|\mathbf{s}_{1}^{H} \mathbf{s}_{1,1}\right|^{2}}{N^{2}}\right) \\
& \approx \frac{N}{\sigma_{n}^{2}} .
\end{aligned}
$$

The assumption made in the last expression (i.e., $\left.\left|\mathbf{s}_{1}^{H} \mathbf{s}_{1,1}\right|^{2} / N^{2} \approx 0\right)$ is very accurate when the signals are not closely spaced. This assumption cannot be verified analytically, as it depends on the structure of the array, however, it can be numerically verified for a commonly used linear equispaced array with half wavelength spacing. The other assumption made throughout this study is that the look direction interferer is above the noise floor (i.e., snr $>1$ ). In this case, we need at least $\left|\beta_{1,1}\right|^{2} \gg 1 / N$ (or equivalently $N\left|\beta_{1,1}\right|^{2}$ snr $\gg 1$ ) in order to detect any multipath power as seen later. We shall also see that when $\left|\beta_{1,1}\right|^{2}$ is closer to the lower bound of $1 / N$ we do not achieve good processing gain to detect multipath unless snr is extremely large (but this case is not analyzed here).

Now we would like to investigate the two cases $\left|\beta_{1,1}\right|^{2} \gg$ $1 / N$ and $\left|\beta_{1,1}\right|^{2} \ll 1 / N$ simultaneously. The value of the expression (A.14) for $\left|\beta_{1,1}\right|^{2} \ll 1 / N$ can be simplified as follow:

$$
\begin{aligned}
\mathbf{s}_{1}^{H} \mathbf{R}_{1}^{-1} \mathbf{s}_{1} & \approx \frac{N}{\sigma_{n}^{2}}\left[1-\frac{\left|\mathbf{s}_{1}^{H} \mathbf{s}_{1,1}\right|^{2}\left|\beta_{1,1}\right|^{2} \mathrm{snr}}{N}\right] \\
& \approx \frac{N}{\sigma_{n}^{2}}\left[1-\frac{\left|\mathbf{s}_{1}^{H} \mathbf{s}_{1,1}\right|^{2}\left(N\left|\beta_{1,1}\right|^{2}\right) \mathrm{snr}}{N^{2}}\right] \\
& \approx \frac{N}{\sigma_{n}^{2}} .
\end{aligned}
$$

Throughout the study, this case is taken to be equivalent to $N\left|\beta_{1,1}\right|^{2}$ snr $\ll 1$ as well because snr is not assumed to take excessively large values for $\left.\left|\beta_{1,1}\right|^{2} \ll 1 / N\right)$. The investigation of the signal processing gain for the case where $\left|\beta_{1,1}\right|^{2} \ll 1 / N$ and at the same time snr is very large is outside the scope of this study.

Furthermore, applying the above formula and (A.11) in (A.3) we can see that

$$
\begin{aligned}
\left|\mathbf{w}_{1}^{H} \mathbf{s}_{1,1}\right|^{2} & =\left|\frac{\mathbf{s}_{1}^{H} \mathbf{R}_{1}^{-1} \mathbf{s}_{1,1}}{\mathbf{s}_{1}^{H} \mathbf{R}_{1}^{-1} \mathbf{s}_{1}}\right|^{2} \\
& =\left|\frac{\mathbf{s}_{1}^{H}}{\mathbf{s}_{1}^{H} \mathbf{R}_{1}^{-1} \mathbf{s}_{1}} \cdot \frac{\mathbf{s}_{1,1}}{\left(\sigma_{n}^{2}+N\left|\beta_{1,1}\right|^{2} \rho_{1}^{2}\right)}\right|^{2} \\
& \approx \frac{\left(\left|\mathbf{s}_{1}^{H} \mathbf{s}_{1,1}\right|^{2} / N^{2}\right)}{\left(1+N\left|\beta_{1,1}\right|^{2} \mathrm{snr}\right)^{2}} \\
& \approx 0 .
\end{aligned}
$$

This expression shows how closely we have achieved the orthogonality requirement expected above. It is reasonable to assume that $\mathbf{w}_{1}^{H} \mathbf{s}_{1,1} \approx 0$ (or equivalently $\left|\mathbf{s}_{1}^{H} \mathbf{s}_{1.1}\right|^{2} / N^{2} \approx$ 0 ) for all possible positive values of $N\left|\beta_{1,1}\right|^{2}$. We may now investigate the second and third terms as the dominant terms at the processor output in (A.8). The approximate expressions for these two terms can be derived using (A.10)(A.14) as follows.

From (A.4) we have

$$
\begin{aligned}
& \beta_{1,1} \mathbf{w}_{2}^{H} \mathbf{s}_{1,1}=\beta_{1,1}[-\beta_{1,1} \rho_{1}^{2} \mathbf{R}_{1}^{-1} \mathbf{s}_{1,1} \\
&\left.+\beta_{1,1} \rho_{1}^{2}\left(\frac{\mathbf{s}_{1}^{H} \mathbf{R}_{1}^{-1} \mathbf{s}_{1,1}}{\mathbf{s}_{1}^{H} \mathbf{R}_{1}^{-1} \mathbf{s}_{1}}\right) \mathbf{R}_{1}^{-1} \mathbf{s}_{1}\right]^{H} \mathbf{s}_{1,1} \\
&=-\left|\beta_{1,1}\right|^{2} \rho_{1}^{2} \mathbf{s}_{1,1}^{H} \mathbf{R}_{1}^{-1} \mathbf{s}_{1,1}+\frac{\left|\beta_{1,1}\right|^{2} \rho_{1}^{2}\left|\mathbf{s}_{1}^{H} \mathbf{R}_{1}^{-1} \mathbf{s}_{1,1}\right|^{2}}{\mathbf{s}_{1}^{H} \mathbf{R}_{1}^{-1} \mathbf{s}_{1}} .
\end{aligned}
$$

Now further simplification of (A.17) using (A.12) leads to

$$
\begin{aligned}
1+\beta_{1,1} \mathbf{w}_{2}^{H} \mathbf{s}_{1,1} \\
=1-\frac{\left|\beta_{1,1}\right|^{2} \rho_{1}^{2} N}{\sigma_{n}^{2}+N\left|\beta_{1,1}\right|^{2} \rho_{1}^{2}}+\frac{\left|\beta_{1,1}\right|^{2} \rho_{1}^{2}\left|\mathbf{s}_{1}^{H} \mathbf{R}_{1}^{-1} \mathbf{s}_{1,1}\right|^{2}}{\mathbf{s}_{1}^{H} \mathbf{R}_{1}^{-1} \mathbf{s}_{1}} \\
=\frac{\sigma_{n}^{2}}{\sigma_{n}^{2}+N\left|\beta_{1,1}\right|^{2} \rho_{1}^{2}}+\frac{\left|\beta_{1,1}\right|^{2} \rho_{1}^{2}\left|\mathbf{s}_{1}^{H} \mathbf{R}_{1}^{-1} \mathbf{s}_{1,1}\right|^{2}}{\mathbf{s}_{1}^{H} \mathbf{R}_{1}^{-1} \mathbf{s}_{1}}
\end{aligned}
$$


where the second term on the right-hand side can be simplified using (A.13), (A.14) and finally assuming $N\left|\beta_{1,1}\right|^{2}$ snr $\gg$ 1 (i.e., $1+N\left|\beta_{1,1}\right|^{2}$ snr $\approx N\left|\beta_{1,1}\right|^{2}$ snr) as follows:

$$
\begin{aligned}
\frac{\left|\beta_{1,1}\right|^{2} \rho_{1}^{2}\left|\mathbf{s}_{1}^{H} \mathbf{R}_{1}^{-1} \mathbf{s}_{1,1}\right|^{2}}{\mathbf{s}_{1}^{H} \mathbf{R}_{1}^{-1} \mathbf{s}_{1}} & =\frac{\left|\beta_{1,1}\right|^{2} \rho_{1}^{2}\left|\mathbf{s}_{1,1}^{H} \mathbf{s}_{1}\right|^{2}}{\left(N / \sigma_{n}^{2}\right)\left(\sigma_{n}^{2}+N\left|\beta_{1,1}\right|^{2} \rho_{1}^{2}\right)^{2}} \\
& =\frac{\left|\beta_{1,1}\right|^{2}\left|\mathbf{s}_{1,1}^{H} \mathbf{s}_{1}\right|^{2} \mathrm{snr}}{N\left(1+N\left|\beta_{1,1}\right|^{2} \mathrm{snr}\right)^{2}} \\
& \approx \frac{\left|\mathbf{s}_{1,1}^{H} \mathbf{s}_{1}\right|^{2} / N^{2}}{\left(N\left|\beta_{1,1}\right|^{2} \mathrm{snr}\right)} \\
& \approx 0 \text { for } N\left|\beta_{1,1}\right|^{2} \text { snr } \gg 1,
\end{aligned}
$$

$$
\begin{aligned}
\frac{\left|\beta_{1,1}\right|^{2} \rho_{1}^{2}\left|\mathbf{s}_{1}^{H} \mathbf{R}_{1}^{-1} \mathbf{s}_{1,1}\right|^{2}}{\mathbf{s}_{1}^{H} \mathbf{R}_{1}^{-1} \mathbf{s}_{1}} & =\frac{\left|\beta_{1,1}\right|^{2}\left|\mathbf{s}_{1,1}^{H} \mathbf{s}_{1}\right|^{2} \mathrm{snr}}{N\left(1+N\left|\beta_{1,1}\right|^{2} \mathrm{snr}\right)^{2}} \\
& \approx \frac{\left|\beta_{1,1}\right|^{2}\left|\mathbf{s}_{1,1}^{H} \mathbf{s}_{1}\right|^{2} \mathrm{snr}}{N} \\
& =\left(N\left|\beta_{1,1}\right|^{2} \mathrm{snr}\right) \frac{\left|\mathbf{s}_{1,1}^{H} \mathbf{s}_{1}\right|^{2}}{N^{2}} \\
& \approx 0 \text { for } N\left|\beta_{1,1}\right|^{2} \mathrm{snr} \ll 1 .
\end{aligned}
$$

As a result we have

$$
\begin{aligned}
\mid 1+ & \left.\beta_{1,1} \mathbf{w}_{2}^{H} \mathbf{s}_{1,1}\right|^{2} \\
& \approx \frac{1}{\left.|1+N| \beta_{1,1}\right|^{2} \text { snr }\left.\right|^{2}} \\
& \approx \frac{1}{\left(N\left|\beta_{1,1}\right|^{2} \text { snr }\right)^{2}} \text { for } N\left|\beta_{1,1}\right|^{2} \text { snr } \gg 1 \\
& \approx 1-2 N\left|\beta_{1,1}\right|^{2} \text { snr } \text { for } N\left|\beta_{1,1}\right|^{2} \text { snr } \ll 1 .
\end{aligned}
$$

The final term of the power output at the processor, that is, $\sigma_{n}^{2}\left(\mathbf{w}_{1}^{H} \mathbf{w}_{1}+\mathbf{w}_{2}^{H} \mathbf{w}_{2}\right)=\sigma_{n}^{2}\|\mathbf{w}\|^{2}$ can be approximated as follows.

Using (A.3) and (A.14) we have

$$
\begin{aligned}
\mathbf{w}_{1}^{H} \mathbf{w}_{1}= & \left(\frac{\mathbf{R}_{1}^{-1} \mathbf{s}_{1}}{\mathbf{s}_{1}^{H} \mathbf{R}_{1}^{-1} \mathbf{s}_{1}}\right)^{H}\left(\frac{\mathbf{R}_{1}^{-1} \mathbf{s}_{1}}{\mathbf{s}_{1}^{H} \mathbf{R}_{1}^{-1} \mathbf{s}_{1}}\right) \\
& \approx \frac{\sigma_{n}^{4}}{N^{2}}\left(\mathbf{R}_{1}^{-1} \mathbf{s}_{1}\right)^{H}\left(\mathbf{R}_{1}^{-1} \mathbf{s}_{1}\right) .
\end{aligned}
$$

Substituting (A.10) and $\boldsymbol{s}_{1}^{H} \mathbf{s}_{1}=N$ in the above expression and noting that $1+N\left|\beta_{1,1}\right|^{2}$ snr $\approx N\left|\beta_{1,1}\right|^{2}$ snr (i.e., $N\left|\beta_{1,1}\right|^{2}$ snr $\gg 1$ ), we get

$$
\begin{aligned}
& \mathbf{w}_{1}^{H} \mathbf{w}_{1} \approx \frac{\sigma_{n}^{4}}{N^{2}} \cdot \frac{1}{\sigma_{n}^{4}}\left\{N-\frac{2 \rho_{1}^{2}\left|\beta_{1,1}\right|^{2}\left|\mathbf{s}_{1}^{H} \mathbf{s}_{1,1}\right|^{2}}{\left(\sigma_{n}^{2}+N\left|\beta_{1,1}\right|^{2} \rho_{1}^{2}\right)}\right. \\
& \left.+\frac{\rho_{1}^{4}\left|\beta_{1,1}\right|^{4}\left|\mathbf{s}_{1}^{H} \mathbf{s}_{1,1}\right|^{2} N}{\left(\sigma_{n}^{2}+N\left|\beta_{1,1}\right|^{2} \rho_{1}^{2}\right)^{2}}\right\} \\
& =\left\{\frac{1}{N}-\frac{2\left|\beta_{1,1}\right|^{2} \operatorname{snr}\left|\mathbf{s}_{1}^{H} \mathbf{s}_{1,1}\right|^{2} / N^{2}}{\left(1+N\left|\beta_{1,1}\right|^{2} \text { snr }\right)}\right. \\
& \left.+\frac{\left|\beta_{1,1}\right|^{4} \mathrm{snr}^{2}\left|\mathbf{s}_{1}^{H} \mathbf{s}_{1,1}\right|^{2} / N}{\left(1+N\left|\beta_{1,1}\right|^{2} \mathrm{snr}\right)^{2}}\right\} \\
& \approx \frac{1}{N}-\frac{\left|\mathbf{s}_{1}^{H} \mathbf{s}_{1,1}\right|^{2}}{N^{3}} \\
& \approx \frac{1}{N} \quad\left(\text { for } N\left|\beta_{1,1}\right|^{2} \text { snr } \gg 1\right) \text {. }
\end{aligned}
$$

For $1+N\left|\beta_{1,1}\right|^{2}$ snr $\approx 1$ (i.e., $N\left|\beta_{1,1}\right|^{2}$ snr $\ll 1$ ) we have

$$
\mathbf{w}_{1}^{H} \mathbf{w}_{1} \approx\left\{\frac{1}{N}-\frac{2\left|\beta_{1,1}\right|^{2} \mathrm{snr}\left|\mathbf{s}_{1}^{H} \mathbf{s}_{1,1}\right|^{2}}{N^{2}}\right.
$$

$$
\begin{gathered}
\left.+\frac{\left|\beta_{1,1}\right|^{4} \mathrm{snr}^{2}\left|\mathbf{s}_{1}^{H} \mathbf{s}_{1,1}\right|^{2}}{N}\right\} \\
=\left\{\frac{1}{N}-\frac{2 N\left|\beta_{1,1}\right|^{2} \operatorname{snr}\left|\mathbf{s}_{1}^{H} \mathbf{s}_{1,1}\right|^{2}}{N^{3}}\right. \\
\left.+\frac{\left(N\left|\beta_{1,1}\right|^{2} \mathrm{snr}\right)^{2}\left|\mathbf{s}_{1}^{H} \mathbf{s}_{1,1}\right|^{2}}{N^{3}}\right\} \\
\approx \frac{1}{N} .
\end{gathered}
$$

From (A.4) we have

$$
\begin{aligned}
\mathbf{w}_{2}^{H} \mathbf{w}_{2}= & \left|\beta_{1,1}\right|^{2} \rho_{1}^{4}\left[-\mathbf{R}_{1}^{-1} \mathbf{s}_{1,1}+\left(\frac{\mathbf{s}_{1}^{H} \mathbf{R}_{1}^{-1} \mathbf{s}_{1,1}}{\mathbf{s}_{1}^{H} \mathbf{R}_{1}^{-1} \mathbf{s}_{1}}\right) \mathbf{R}_{1}^{-1} \mathbf{s}_{1}\right]^{H} \\
& \times\left[-\mathbf{R}_{1}^{-1} \mathbf{s}_{1,1}+\left(\frac{\mathbf{s}_{1}^{H} \mathbf{R}_{1}^{-1} \mathbf{s}_{1,1}}{\mathbf{s}_{1}^{H} \mathbf{R}_{1}^{-1} \mathbf{s}_{1}}\right) \mathbf{R}_{1}^{-1} \mathbf{s}_{1}\right] .
\end{aligned}
$$


The dominant term in the expression for $\mathbf{w}_{2}^{H} \mathbf{w}_{2}$ is given by the first term inside the bracket involving $\mathbf{R}_{1}^{-1} \mathbf{s}_{1,1}$, which can be simplified using (A.11) as

$$
\begin{aligned}
\mathbf{w}_{2}^{H} \mathbf{w}_{2} & \approx\left|\beta_{1,1}\right|^{2} \rho_{1}^{4}\left(\mathbf{R}_{1}^{-1} \mathbf{s}_{1,1}\right)^{H}\left(\mathbf{R}_{1}^{-1} \mathbf{s}_{1,1}\right) \\
& =\frac{\left|\beta_{1,1}\right|^{2} \rho_{1}^{4} N}{\left(\sigma_{n}^{2}+N\left|\beta_{1,1}\right|^{2} \rho_{1}^{2}\right)^{2}} \\
& =\frac{\left|\beta_{1,1}\right|^{2} N s r^{2}}{\left(1+N\left|\beta_{1,1}\right|^{2} \mathrm{snr}\right)^{2}} \\
& \approx \frac{1}{N\left|\beta_{1,1}\right|^{2}}
\end{aligned}
$$

for $N\left|\beta_{1,1}\right|^{2}$ snr $\gg 1$.

The final expression is

$$
\mathbf{w}_{2}^{H} \mathbf{w}_{2} \approx \begin{cases}\frac{1}{N\left|\beta_{1,1}\right|^{2}} & \text { for } N\left|\beta_{1,1}\right|^{2} \text { snr } \gg 1, \\ N\left|\beta_{1,1}\right|^{2} \text { snr }^{2} & \text { for } N\left|\beta_{1,1}\right|^{2} \text { snr } \ll 1 .\end{cases}
$$

We can show that the contributions arising from the three other terms in (A.25) are negligible as follows. The second term in the brackets of (A.25) contains the term $\left(\mathbf{s}_{1}^{H} \mathbf{R}_{1}^{-1} \mathbf{s}_{1,1} / \mathbf{s}_{1}^{H} \mathbf{R}_{1}^{-1} \mathbf{s}_{1}\right) \mathbf{R}_{1}^{-1} \mathbf{s}_{1}$, the square of which after substituting (A.13) and (A.14) takes the following form:

$$
\begin{aligned}
\left|\beta_{1,1}\right|^{2} \rho_{1}^{4}\left|\frac{\mathbf{s}_{1}^{H} \mathbf{R}_{1}^{-1} \mathbf{s}_{1,1}}{\mathbf{s}_{1}^{H} \mathbf{R}_{1}^{-1} \mathbf{s}_{1}}\right|^{2}\left\|\mathbf{R}_{1}^{-1} \mathbf{s}_{1}\right\|^{2} \\
=\frac{\left|\beta_{1,1}\right|^{2} \sigma_{n}^{4} \rho_{1}^{4}\left|\mathbf{s}_{1}^{H} \mathbf{s}_{1,1}\right|^{2} / N^{2}}{\left(\sigma_{n}^{2}+N\left|\beta_{1,1}\right|^{2} \rho_{1}^{2}\right)^{2}}\left\|\mathbf{R}_{1}^{-1} \mathbf{s}_{1}\right\|^{2},
\end{aligned}
$$

where (from (A.10))

$$
\begin{aligned}
\|\left.\mathbf{R}_{1}^{-1} \mathbf{s}_{1}\right|^{2}= & \frac{1}{\sigma_{n}^{4}}\left(\mathbf{s}_{1}-\frac{\rho_{1}^{2}\left|\beta_{1,1}\right|^{2} \mathbf{s}_{1,1} \mathbf{s}_{1,1}^{H} \mathbf{s}_{1}}{\sigma_{n}^{2}+N\left|\beta_{1,1}\right|^{2} \rho_{1}^{2}}\right)^{H} \\
& \times\left(\mathbf{s}_{1}-\frac{\rho_{J}^{2}\left|\beta_{1,1}\right|^{2} \mathbf{s}_{1,1} \mathbf{s}_{1,1}^{H} \mathbf{s}_{1}}{\sigma_{n}^{2}+N\left|\beta_{1,1}\right|^{2} \rho_{1}^{2}}\right) \\
= & \frac{1}{\sigma_{n}^{4}}\left(N-\frac{2\left|\beta_{1,1}\right|^{2} \mathrm{snr}\left|\mathbf{s}_{1,1}^{H} \mathbf{s}_{1}\right|^{2}}{1+N\left|\beta_{1,1}\right|^{2} \mathrm{snr}}\right. \\
& \left.+\frac{\left|\beta_{1,1}\right|^{4} N \mathrm{snr}^{2}\left|\mathbf{s}_{1,1}^{H} \mathbf{s}_{1}\right|^{2}}{\left(1+N\left|\beta_{1,1}\right|^{2} \mathrm{snr}\right)^{2}}\right)
\end{aligned}
$$

Simplifying the above expression and finally substituting $1+$ $N\left|\beta_{1,1}\right|^{2}$ snr $\approx N\left|\beta_{1,1}\right|^{2}$ snr we have $\left\|\mathbf{R}_{1}^{-1} \mathbf{s}_{1}\right\|^{2} \approx\left(N / \sigma_{n}^{4}\right)(1-$
$\left.\left|\mathbf{s}_{1}^{H} \mathbf{s}_{1,1}\right|^{2} / N^{2}\right) \approx N / \sigma_{n}^{4}$. On the other hand for $N\left|\beta_{1,1}\right|^{2}$ snr $\ll$ 1 we have

$$
\begin{aligned}
\|\left.\mathbf{R}_{1}^{-1} \mathbf{s}_{1}\right|^{2} \approx & \frac{N}{\sigma_{n}^{4}}\left(1-\frac{2\left|\beta_{1,1}\right|^{2}\left|\mathbf{s}_{1}^{H} \mathbf{s}_{1,1}\right|^{2} \mathrm{snr}}{N}\right. \\
& \left.+\left|\beta_{1,1}\right|^{4}\left|\mathbf{s}_{1}^{H} \mathbf{s}_{1,1}\right|^{2} \mathrm{snr}^{2}\right) \\
\approx & \frac{N}{\sigma_{n}^{4}}\left(1-\frac{2\left(N\left|\beta_{1,1}\right|^{2} \mathrm{snr}\right)\left|\mathbf{s}_{1}^{H} \mathbf{s}_{1,1}\right|^{2}}{N^{2}}\right. \\
& \left.+\frac{\left(N\left|\beta_{1,1}\right|^{2} \mathrm{snr}\right)^{2}\left|\mathbf{s}_{1}^{H} \mathbf{s}_{1,1}\right|^{2}}{N^{2}}\right) \\
\approx & \frac{N}{\sigma_{n}^{4}} .
\end{aligned}
$$

Back substitution of these expressions in (A.28) and the use of $1+N\left|\beta_{1,1}\right|^{2}$ snr $\approx N\left|\beta_{1,1}\right|^{2}$ snr lead to the following expression:

$$
\begin{aligned}
& \left|\beta_{1,1}\right|^{2} \rho_{1}^{4}\left|\frac{\mathbf{s}_{1}^{H} \mathbf{R}_{1}^{-1} \mathbf{s}_{1,1}}{\mathbf{s}_{1}^{H} \mathbf{R}_{1}^{-1} \mathbf{s}_{1}}\right|^{2} \|\left.\mathbf{R}_{1}^{-1} \mathbf{s}_{1}\right|^{2} \\
& =\frac{\left|\beta_{1,1}\right|^{2} \mathrm{snr}^{2}\left|\mathbf{s}_{1}^{H} \mathbf{s}_{1,1}\right|^{2}}{N\left(1+N\left|\beta_{1,1}\right|^{2} \text { snr }\right)^{2}} \\
& \approx \frac{\left|\mathbf{s}_{1}^{H} \mathbf{s}_{1,1}\right|^{2} / N^{2}}{N\left|\beta_{1,1}\right|^{2}} \\
& \approx 0 \quad \text { for } N\left|\beta_{1,1}\right|^{2} \text { snr } \gg 1,
\end{aligned}
$$

and for $N\left|\beta_{1,1}\right|^{2}$ snr $\ll 1$ we have

$$
\begin{aligned}
\frac{\left|\beta_{1,1}\right|^{2} \mathrm{snr}^{2}\left|\mathbf{s}_{1}^{H} \mathbf{s}_{1,1}\right|^{2}}{N\left(1+N\left|\beta_{1,1}\right|^{2} \text { snr }\right)^{2}} & \approx \frac{\left|\beta_{1,1}\right|^{2} \operatorname{snr}^{2}\left|\mathbf{s}_{1}^{H} \mathbf{s}_{1,1}\right|^{2}}{N} \\
& \approx\left(N\left|\beta_{1,1}\right|^{2} \operatorname{snr}^{2}\right)\left(\frac{\left|\mathbf{s}_{1}^{H} \mathbf{s}_{1,1}\right|^{2}}{N^{2}}\right) \\
& \approx 0 .
\end{aligned}
$$

The third contribution in (A.25) is given by (sum of two terms)

$$
\begin{gathered}
-2 \text { Real }\left\{\frac{\rho_{1}^{4}\left|\beta_{1,1}\right|^{2}\left(\mathbf{s}_{1,1}^{H} \mathbf{R}_{1}^{-1} \mathbf{s}_{1}\right)\left(\mathbf{s}_{1}^{H} \mathbf{R}_{1}^{-1} \mathbf{R}_{1}^{-1} \mathbf{s}_{1,1}\right)}{\left(\mathbf{s}_{1}^{H} \mathbf{R}_{1}^{-1} \mathbf{s}_{1}\right)}\right\} \\
=\frac{2\left|\beta_{1,1}\right|^{2} \mathrm{snr}^{2}\left|\mathbf{s}_{1,1}^{H} \mathbf{s}_{1}\right|^{2}}{N\left(1+N\left|\beta_{1,1}\right|^{2} \mathrm{snr}\right)^{3}} .
\end{gathered}
$$

(Note: replacing $\left(\mathbf{s}_{1}^{H} \mathbf{R}_{1}^{-1} \mathbf{s}_{1}\right)$ by the approximation $N / \sigma_{n}^{2}$ and the use of (A.10), (A.11) and (A.13) in (A.33), we arrive at the expression in the right-hand side of (A.33)). In fact after applying the approximation $1+N\left|\beta_{1,1}\right|^{2} \mathrm{snr} \approx N\left|\beta_{1,1}\right|^{2} \mathrm{snr}$ 
or $1+N\left|\beta_{1,1}\right|^{2}$ snr $\approx 1$ we can conclude that the right-hand side of (A.33) is approximately equal to zero. From (A.5) and (A.27), the final expression for $\sigma_{n}^{2}\|\mathbf{w}\|^{2}$ given by (combining (A.23) and (A.27))

$\sigma_{n}^{2}\|\mathbf{w}\|^{2}= \begin{cases}\sigma_{n}^{2}\left(\frac{1}{N}+\frac{1}{N\left|\beta_{1,1}\right|^{2}}\right) & \text { for } N\left|\beta_{1,1}\right|^{2} \text { snr } \gg 1, \\ \sigma_{n}^{2}\left(\frac{1}{N}+N\left|\beta_{1,1}\right|^{2} \operatorname{snr}^{2}\right) & \text { for } N\left|\beta_{1,1}\right|^{2} \text { sn } \ll 1 .\end{cases}$

Now we have

$$
\begin{aligned}
& \left|1+\beta_{1,1} \mathbf{w}_{2}^{H} \mathbf{s}_{1,1}\right|^{2} \approx \frac{1}{\left(N\left|\beta_{1,1}\right|^{2} \text { snr }\right)^{2}} \text { for } N\left|\beta_{1,1}\right|^{2} \text { snr } \gg 1 \\
& \approx 1-2 N\left|\beta_{1,1}\right|^{2} \text { snr } \quad \text { for } N\left|\beta_{1,1}\right|^{2} \text { snr } \ll 1 . \\
& \sigma_{n}^{2}\|\mathbf{w}\|^{2}= \begin{cases}\sigma_{n}^{2}\left(\frac{1}{N}+\frac{1}{N\left|\beta_{1,1}\right|^{2}}\right) & \text { for } N\left|\beta_{1,1}\right|^{2} \operatorname{snr} \gg 1 \\
\sigma_{n}^{2}\left(\frac{1}{N}+N\left|\beta_{1,1}\right|^{2} \operatorname{snr}^{2}\right) & \text { for } N\left|\beta_{1,1}\right|^{2} \operatorname{snr} \ll 1 .\end{cases}
\end{aligned}
$$

Substituting (A.35) in (A.8) we can evaluate $P_{\text {out }} / \sigma_{n}^{2}$ as

$$
\frac{P_{\text {out }}}{\sigma_{n}^{2}} \approx\left\{\begin{array}{c}
\left(\frac{1}{N}+\frac{1}{N\left|\beta_{1,1}\right|^{2}}\right)+\frac{1}{N^{2}\left|\beta_{1,1}\right|^{4} \text { snr }}, \\
N\left|\beta_{1,1}\right|^{2} \text { snr } \gg 1 \\
\frac{1}{N}+\text { snr }-N\left|\beta_{1,1}\right|^{2} \text { snr }^{2}, \\
N\left|\beta_{1,1}\right|^{2} \text { snr } \ll 1,
\end{array}\right.
$$

which becomes

$$
\frac{P_{\text {out }}}{\sigma_{n}^{2}} \approx \begin{cases}\frac{N\left|\beta_{1,1}\right|^{4} \text { snr }+N\left|\beta_{1,1}\right|^{2} \text { snr }+1}{N^{2}\left|\beta_{1,1}\right|^{4} \text { snr }}, & N\left|\beta_{1,1}\right|^{2} \text { snr } \gg 1 \\ \frac{1}{N}+\text { snr }, & N\left|\beta_{1,1}\right|^{2} \text { snr } \ll 1 .\end{cases}
$$

After substituting $N\left|\beta_{1,1}\right|^{4}$ snr $+N\left|\beta_{1,1}\right|^{2}$ snr $+1 \approx$ $N\left|\beta_{1,1}\right|^{4}$ snr $+N\left|\beta_{1,1}\right|^{2}$ snr in the above expression for $N\left|\beta_{1,1}\right|^{2}$ snr $\gg 1$ case, we have

$$
\frac{\sigma_{n}^{2}}{P_{\text {out }}} \approx \begin{cases}\frac{N\left|\beta_{1,1}\right|^{2}}{1+\left|\beta_{1,1}\right|^{2}}, & N\left|\beta_{1,1}\right|^{2} \text { snr } \gg 1 \\ \frac{N}{1+N \text { snr }-N^{2}\left|\beta_{1,1}\right|^{2} \text { snr }^{2}}, & N\left|\beta_{1,1}\right|^{2} \text { snr } \ll 1 .\end{cases}
$$

As seen later in the simulation section, the conclusions drawn here do not change significantly when one or two sidelobe interferers (other sources) are considered. The only difference is that (A.8) will have additional terms due to sidelobe interferers and other multipaths. The added terms in (A.8) are of the form $\rho_{k}^{2}\left|\mathbf{w}_{1}^{H} \mathbf{s}_{k}\right|^{2}(k=1,2, \ldots)$ and they should satisfy the orthogonality requirement in a very similar manner. By denoting the value of $T_{S}(n)$ for $n=n_{1,1}$ by $T_{S}(n)_{n=n_{1,1}}$ we can use the result in (A.38) and the identity obtained in Appendix A to further simplify (6) to show that $T_{S}(n)_{n=n_{1,1}}$

$$
=\left\{\begin{array}{l}
\left(\rho_{1}^{2}+\left(\mathbf{s}_{1}^{H} \mathbf{R}_{1}^{-1} \mathbf{s}_{1}\right)^{-1}\right) \frac{N\left|\beta_{1,1}\right|^{2}}{\sigma_{n}^{2}\left(1+\left|\beta_{1,1}\right|^{2}\right)}-1, \\
N\left|\beta_{1,1}\right|^{2} \text { snr } \gg 1 \\
\left(\rho_{1}^{2}+\left(\mathbf{s}_{1}^{H} \mathbf{R}_{1}^{-1} \mathbf{s}_{1}\right)^{-1}\right) \frac{N}{\sigma_{n}^{2}\left(1+N \operatorname{snr}-N^{2}\left|\beta_{1,1}\right|^{2} \mathrm{snr}^{2}\right)}-1, \\
N\left|\beta_{1,1}\right|^{2} \text { snr } \ll 1 .
\end{array}\right.
$$

For the case of a small number of sources and multipaths we have shown that $\left(\mathbf{s}_{1}^{H} \mathbf{R}_{1}^{-1} \mathbf{s}_{1}\right) \approx N / \sigma_{n}^{2}$ for $N\left|\beta_{1,1}\right|^{2}$ snr $\ll 1$ and $N\left|\beta_{1,1}\right|^{2}$ snr $\gg 1$. As a result we have for $N\left|\beta_{1,1}\right|^{2}$ snr $\gg 1$

$$
\begin{aligned}
T_{S}(m)_{m=n_{1,1}} & =\left(\rho_{1}^{2}+\frac{\sigma_{n}^{2}}{N}\right) \frac{N\left|\beta_{1,1}\right|^{2}}{\sigma_{n}^{2}\left(1+\left|\beta_{1,1}\right|^{2}\right)}-1 \\
& \approx \frac{N\left|\beta_{1,1}\right|^{2} \mathrm{snr}-1}{\left(1+\left|\beta_{1,1}\right|^{2}\right)} \\
& \approx N\left|\beta_{1,1}\right|^{2} \mathrm{snr},
\end{aligned}
$$

and for $N\left|\beta_{1,1}\right|^{2}$ snr $\ll 1$

$$
\begin{aligned}
& T_{S}(n)_{n=n_{1,1}} \\
&=\frac{\left(\rho_{1}^{2}+\sigma_{n}^{2} / N\right)}{P_{\text {out }}} \\
&-1 \frac{N\left(\rho_{1}^{2}+\sigma_{n}^{2} / N\right)}{\sigma_{n}^{2}\left(1+N \text { snr }-N^{2}\left|\beta_{1,1}\right|^{2}\right.} \text { sn } \\
& \approx \frac{N^{2}\left|\beta_{1,1}\right|^{2} \mathrm{snr}^{2}}{\left(1+N \operatorname{snr}\left(1-N\left|\beta_{1,1}\right|^{2} \mathrm{snr}\right)\right)} \\
& \approx \frac{N^{2}\left|\beta_{1,1}\right|^{2} \mathrm{snr}^{2}}{\left(1+N_{\mathrm{snr}}\right)} \\
& \approx N\left|\beta_{1,1}\right|^{2} \mathrm{snr} .
\end{aligned}
$$$$
-1 \frac{N\left(\rho_{1}^{2}+\sigma_{n}^{2} / N\right)}{\sigma_{n}^{2}\left(1+N \operatorname{snr}-N^{2}\left|\beta_{1,1}\right|^{2} \operatorname{snr}^{2}\right)}-1
$$

The TSI finder spectrum has the following properties:

$$
T_{S}(n)= \begin{cases}N\left|\beta_{1,1}\right|^{2} \text { snr, } & n=n_{1,1}, \\ 0, & n \neq n_{1,1} .\end{cases}
$$

In order to quantify the processing gain of this spectrum one has to replace the zero figure with a quantity which 
would represent the average output interference level present in the spectrum whenever a lag mismatch occurs. Replacing $\mathbf{Q}^{H}=\mathbf{O}_{N \times N}$ in (6) by an approximate figure (when $n \neq n_{1,1}$ ) would give rise to a small nonzero value. This figure can be shown to be of the order $N / M \operatorname{snr}$ (written as $O(N / M s n r)$ ), where $M$ is the number of samples used in covariance averaging. As a result we can establish processing gain as

$$
\frac{T_{S}(n)_{n=n_{1,1}}}{T_{S}(n)_{n \neq n_{1,1}}} \approx \frac{N\left|\beta_{1,1}\right|^{2} s n r}{O(N / M s n r))} \approx O\left(M\left|\beta_{1,1}\right|^{2} \text { snr }^{2}\right) .
$$

The above expression can be derived using the following argument. Consider the case when $n \neq n_{1,1}$, but $\beta_{1,1} \approx 0$. In this case we have

$$
\begin{aligned}
\left(\begin{array}{c}
\mathbf{x}(r) \\
\mathbf{x}(t+n)
\end{array}\right) & =\left(\begin{array}{c}
j_{1}(t) \mathbf{s}_{1}+\boldsymbol{\varepsilon}_{1} \\
j_{1}(t+n) \mathbf{s}_{1}+\boldsymbol{\varepsilon}_{2}
\end{array}\right) \\
\mathbf{R} & =\left(\begin{array}{cc}
\mathbf{R}_{x} & \mathbf{Q}^{H} \\
\mathbf{Q} & \mathbf{R}_{x}
\end{array}\right),
\end{aligned}
$$

where

$$
\begin{aligned}
\mathbf{Q}^{H}= & E\left\{j_{1}(t) j_{1}(r+n)^{*}\right\} \mathbf{s}_{1} \mathbf{s}_{1}^{H}+E\left\{j_{1}(t) \mathbf{s}_{1} \boldsymbol{\varepsilon}_{2}^{H}\right\} \\
& \left.\left.+E\left\{j_{1}(t+n)^{*} \boldsymbol{\varepsilon}_{1} \mathbf{s}_{1}^{H}\right\}+E\right\} \boldsymbol{\varepsilon}_{1} \boldsymbol{\varepsilon}_{2}^{H}\right\} .
\end{aligned}
$$

Generally this term is zero when a large sample support is available for estimating the covariance matrix. However, we would like to estimate the order of the next term as a function of $M$ (number of samples) for large $M$. Suppose $X$ and $Y$ are two independent complex random variables with zero mean and Gaussian distribution then $E\left\{X Y^{*}\right\}=0$, but the estimator would be $Z=(1 / M) \sum_{i=1}^{M} x_{i} y_{i}^{*}$, where $x_{i}$ and $y_{i}$ are the measured sample values. The variance of the estimator is given by $\operatorname{Var}\{Z\}=E\left\{|Z|^{2}\right\}=(1 / M) \sigma_{x}^{2} \sigma_{y}^{2}$, where $\sigma_{x}^{2}$ and $\sigma_{y}^{2}$ are the respective individual variances. As a result we may approximately take the error term to be in the order $(1 / \sqrt{M}) \sigma_{x} \sigma_{y}$ (one standard deviation off the mean value) and represent by $O\left(\sigma_{x} \sigma_{y} / \sqrt{M}\right)$. Now we may consider the following approximate representations:

$$
\begin{aligned}
& E\left\{j_{1}(t) j_{1}(r+n)^{*} \mathbf{s}_{1} \mathbf{s}_{1}^{H}\right\} \approx O\left(\frac{\rho_{1}^{2} \mathbf{s}_{1} \mathbf{s}_{1}^{H}}{\sqrt{M}}\right), \\
& E\left\{j_{1}(t) \mathbf{s}_{1} \boldsymbol{\varepsilon}_{2}^{H}\right\} \approx O\left(\frac{\rho_{1} \sigma_{n} \mathbf{s}_{1} \mathbf{u}^{H}}{\sqrt{M}}\right), \\
& E\left\{j_{1}(t) \boldsymbol{\varepsilon}_{1} \mathbf{s}_{1}^{H}\right\} \approx O\left(\frac{\rho_{1} \sigma_{n} \mathbf{u} \mathbf{s}_{1}^{H}}{\sqrt{M}}\right), \\
& E\left\{\boldsymbol{\varepsilon}_{1} \boldsymbol{\varepsilon}_{2}^{H}\right\} \approx O\left(\frac{\sigma_{n}^{2} \mathbf{u} \mathbf{u}^{H}}{\sqrt{M}}\right)
\end{aligned}
$$

(this term will be ignored as a lower-order term when $\rho_{1}^{2}>$ $\left.\sigma_{n}^{2}\right)$, where $\mathbf{u}=(1,1, \ldots, 1)^{T}$. Noting that $\mathbf{R}_{x}=\mathbf{R}_{1}+\rho_{1}^{2} \mathbf{s}_{1} \mathbf{s}_{1}^{H}$ and $\mathbf{R}_{1}=\sigma_{n}^{2} \mathbf{I}_{N}\left(\right.$ for $\left.\beta_{1,1}=0\right)$, we have

$$
\begin{aligned}
P_{\text {out }}= & \mathbf{w}^{H} E\left\{\mathbf{x}(t) \mathbf{x}(t+n)^{H}\right\} \mathbf{w} \\
= & \mathbf{w}_{1}^{H} \mathbf{R}_{x} \mathbf{w}_{1}+\mathbf{w}_{2}^{H} \mathbf{R}_{x} \mathbf{w}_{2} \\
& +\frac{O\left(\mathbf{w}_{1}^{H}\left(\rho_{1}^{2} \mathbf{s}_{1} \mathbf{s}_{1}^{H}+\rho_{1} \sigma_{n} \mathbf{s}_{1} \mathbf{u}^{H}+\rho_{1} \sigma_{n} \mathbf{u} \mathbf{s}_{1}^{H}\right) \mathbf{w}_{2}\right)}{\sqrt{M}} \\
& +\frac{O\left(\mathbf{w}_{2}^{H}\left(\rho_{1}^{2} \mathbf{s}_{1} \mathbf{s}_{1}^{H}+\rho_{1} \sigma_{n} \mathbf{u} \mathbf{s}_{1}^{H}+\rho_{1} \sigma_{n} \mathbf{s}_{1} \mathbf{u}^{H}\right) \mathbf{w}_{1}\right)}{\sqrt{M}} .
\end{aligned}
$$

Now considering the requirements in the minimization problem (i.e., $\mathbf{w}_{1}^{H} \mathbf{s}_{1}=1$ and $\mathbf{w}_{2}^{H} \mathbf{s}_{1}=0$ ) we have to minimize

$$
\begin{aligned}
P_{\text {out }}= & \rho_{1}^{2}+\mathbf{w}_{1}^{H} \mathbf{R}_{1} \mathbf{w}_{1}+\mathbf{w}_{2}^{H} \mathbf{R}_{1} \mathbf{w}_{2} \\
& +O\left(\frac{\rho_{1} \sigma_{n}\left(\mathbf{u}^{H} \mathbf{w}_{2}+\mathbf{w}_{2}^{H} \mathbf{u}\right)}{\sqrt{M}}\right) .
\end{aligned}
$$

The solution for $\mathbf{w}_{1}$ (which minimizes $P_{\text {out }}$, subject to) is given by

$$
\mathbf{w}_{1}=\frac{\mathbf{R}_{1}^{-1} \mathbf{s}_{1}}{\left(\mathbf{s}_{1}^{H} \mathbf{R}_{1}^{-1} \mathbf{s}_{1}\right)}=\frac{\mathbf{s}_{1}}{N} \quad\left(\text { for } \mathbf{R}_{1}=\sigma_{n}^{2} \mathbf{I}_{N}\right),
$$

and the solution for $\mathbf{w}_{2}$ is given by minimizing $\mathbf{w}_{2}^{H} \mathbf{R}_{1} \mathbf{w}_{2}+$ $O\left(\rho_{1} \sigma_{n}\left(\mathbf{u}^{H} \mathbf{w}_{2}+\mathbf{w}_{2}^{H} \mathbf{u}\right) / \sqrt{M}\right)$ (subject to $\mathbf{w}_{2}^{H} \mathbf{s}_{1}=0$ ). This leads to $\mathbf{w}_{2}=-O\left(\rho_{1} \sigma_{n} \mathbf{R}_{1}^{-1} \mathbf{u} / \sqrt{M}\right)+\mu \mathbf{R}_{1}^{-1} \mathbf{s}_{1}$, where $\mu$ is a constant. Now substituting $\mathbf{w}_{2}^{H} \mathbf{s}_{1}=0$, we have $\mu^{*}=$ $O\left(\rho_{1} \sigma_{n}\left(\mathbf{u}^{H} \mathbf{R}_{1}^{-1} \mathbf{s}_{1}\right) /\left(\left(\mathbf{s}_{1}^{H} \mathbf{R}_{1}^{-1} \mathbf{s}_{1}\right) / \sqrt{M}\right)\right)$. As result we have

$$
\mathbf{w}_{2}=O\left(\left(\frac{\rho_{1} \sigma_{n}}{\sqrt{M}}\right) \mathbf{R}_{1}^{-1} \mathbf{u}-\left(\frac{\rho_{1} \sigma_{n}}{\sqrt{M}}\right)\left(\frac{\left(\mathbf{s}_{1}^{H} \mathbf{R}_{1}^{-1} \mathbf{u}\right)}{\left(\mathbf{s}_{1}^{H} \mathbf{R}_{1}^{-1} \mathbf{s}_{1}\right)}\right) \mathbf{R}_{1}^{-1} \mathbf{s}_{1}\right),
$$

and for $\mathbf{R}_{1}=\sigma_{n}^{2} \mathbf{I}_{N}$ this reduces to $\mathbf{w}_{2}=O\left(\left(\rho_{1} / \sigma_{n}\right)(\mathbf{u} / \sqrt{M})+\right.$ $\left.\left(\left(\rho_{1} / \sigma_{n}\right)\left(\mathbf{s}_{1}^{H} \mathbf{u} / N\right)\left(\mathbf{s}_{1} / \sqrt{M}\right)\right)\right)$. Since $\left(\mathbf{s}_{1}^{H} \mathbf{u} / N\right)<1$, it is reasonable to ignore the low-order term in $\mathbf{w}_{2}$ to take the dominant term only and write $\mathbf{w}_{2} \approx O\left(\left(\rho_{1} / \sigma_{n}\right)(\mathbf{u} / \sqrt{M})\right)$ (substituting $\mathbf{R}_{1}=\sigma_{n}^{2} \mathbf{I}_{N}$ as well as $\mathbf{u}^{H} \mathbf{u}=N$ in $\left.P_{\text {out }}\right)$

$P_{\text {out }} \approx \rho_{1}^{2}+\sigma_{n}^{2} / N+O\left(\rho_{1}^{2} N / M\right)$. Therefore when $\beta_{1,1} \approx 0$ (i.e., no significant multipath energy is available at the lag of interest) we may use (6) and (B.4) to approximate the following:

$$
\begin{aligned}
T(n)_{n \neq n_{1,1}} & \approx \frac{\rho_{1}^{2}+\left(\mathbf{s}_{1}^{H} \mathbf{R}_{1}^{-1} \mathbf{s}_{1}\right)^{-1}}{\rho_{1}^{2}+\sigma_{n}^{2} / N+O\left(\rho_{1}^{2} N / M\right)}-1 \\
& \approx \frac{\rho_{1}^{2}+\sigma_{n}^{2} / N-\left(\rho_{1}^{2}+\sigma_{n}^{2} / N+O\left(\sigma_{1}^{2} N / M\right)\right)}{\rho_{1}^{2}+\sigma_{n}^{2} / N+O\left(\rho_{1}^{2} N / M\right)} \\
& \approx \frac{O\left(\rho_{1}^{2} N / M\right)}{\rho_{1}^{2}} \\
& \approx O\left(\frac{N}{M}\right) .
\end{aligned}
$$


Now we investigate a mismatch with a TSI energy present $\left(\left|\beta_{1,1}\right|^{2} \gg 1 / N\right)$.

Terms involved in $\mathbf{Q}^{H}$ are given by

$$
\begin{aligned}
\mathbf{Q}^{H}=\{ & \left(j_{1}(t) \mathbf{s}_{1}+\beta_{1,1} j_{1}\left(t-n_{1,1}\right) \mathbf{s}_{1,1}+\boldsymbol{\varepsilon}_{1}\right) \\
& \left.\times\left(j_{1}(t+n)^{*} \mathbf{s}_{1}^{H}+\beta_{1,1}^{*} j_{1}\left(t-n_{1,1}+n\right)^{*} \mathbf{s}_{1,1}^{H}+\boldsymbol{\varepsilon}_{2}^{H}\right)\right\} .
\end{aligned}
$$

This can be represented by

$$
\begin{aligned}
\mathbf{Q}^{H} \approx O & \left(\frac{\rho_{1}^{2} \mathbf{s}_{1} \mathbf{s}_{1}^{H}}{\sqrt{M}}, \frac{\left|\beta_{1,1}\right|^{2} \rho_{1}^{2} \mathbf{s}_{1,1} \mathbf{s}_{1,1}^{H}}{\sqrt{M}}, \frac{\sigma_{n}^{2} \mathbf{u} \mathbf{u}^{H}}{\sqrt{M}},\right. \\
& \frac{\beta_{1,1}^{*} \rho_{1}^{2} \mathbf{s}_{1} \mathbf{s}_{1,1}^{H}}{\sqrt{M}}, \frac{\rho_{1} \sigma_{n} \mathbf{s}_{1} \mathbf{u}^{H}}{\sqrt{M}}, \frac{\beta_{1,1} \rho_{1}^{2} \mathbf{s}_{1,1} \mathbf{s}_{1}^{H}}{\sqrt{M}}, \\
& \left.\frac{\beta_{1,1} \rho_{1} \sigma_{n} \mathbf{s}_{1,1} \mathbf{u}^{H}}{\sqrt{M}}, \frac{\rho_{1} \sigma_{n} \mathbf{u} \mathbf{s}_{1}^{H}}{\sqrt{M}}, \frac{\beta_{1,1}^{*} \rho_{1} \sigma_{n} \mathbf{u} \mathbf{s}_{1,1}^{H}}{\sqrt{M}}\right) .
\end{aligned}
$$

In $P_{\text {out }}=\mathbf{w}^{H} \mathbf{R}_{2} \mathbf{w}$, the contribution due to the presence of nonzero $\mathbf{Q}$ is given by the term $\mathbf{w}_{1}^{H} \mathbf{Q}^{H} \mathbf{w}_{2}+\mathbf{w}_{2}^{H} \mathbf{Q} \mathbf{w}_{1}$. This is equivalent to the terms (all positive contributions)

$$
\begin{gathered}
\frac{\rho_{1}^{2}\left(\mathbf{w}_{1}^{H} \mathbf{s}_{1} \mathbf{s}_{1}^{H} \mathbf{w}_{2}+\mathbf{w}_{2}^{H} \mathbf{s}_{1} \mathbf{s}_{1}^{H} \mathbf{w}_{1}\right)}{\sqrt{M}}, \\
\frac{\left|\beta_{1,1}\right|^{2} \rho_{1}^{2}\left(\mathbf{w}_{1}^{H} \mathbf{s}_{1,1} \mathbf{s}_{1,1}^{H} \mathbf{w}_{2}+\mathbf{w}_{2}^{H} \mathbf{s}_{1,1} \mathbf{s}_{1,1}^{H} \mathbf{w}_{1}\right)}{\sqrt{M}}, \\
\frac{\sigma_{n}^{2}\left(\mathbf{w}_{1}^{H} \mathbf{u} \mathbf{u}^{H} \mathbf{w}_{2}+\mathbf{w}_{2}^{H} \mathbf{u} \mathbf{u}^{H} \mathbf{w}_{1}\right)}{\sqrt{M}}, \\
\frac{\rho_{1}^{2}\left(\beta_{1,1}^{*} \mathbf{w}_{1}^{H} \mathbf{s}_{1} \mathbf{s}_{1,1}^{H} \mathbf{w}_{2}+\beta_{1,1} \mathbf{w}_{2}^{H} \mathbf{s}_{11} \mathbf{s}_{1}^{H} \mathbf{w}_{1}\right)}{\sqrt{M}}, \\
\frac{\rho_{1} \sigma_{n}\left(\mathbf{w}_{1}^{H} \mathbf{s}_{1} \mathbf{u}^{H} \mathbf{w}_{2}+\mathbf{w}_{2}^{H} \mathbf{u} \mathbf{s}_{1}^{H} \mathbf{w}_{1}\right)}{\sqrt{M}}, \\
\frac{\rho_{1}^{2}\left(\beta_{1,1} \mathbf{w}_{1}^{H} \mathbf{s}_{1,1} \mathbf{s}_{1}^{H} \mathbf{w}_{2}+\beta_{1,1}^{*} \mathbf{w}_{2}^{H} \mathbf{s}_{1} \mathbf{s}_{1,1}^{H} \mathbf{w}_{1}\right)}{\sqrt{M}} \\
\frac{\rho_{1} \sigma_{n}\left(\beta_{1,1} \mathbf{w}_{1}^{H} \mathbf{s}_{1,1} \mathbf{u}^{H} \mathbf{w}_{2}+\beta_{1,1}^{*} \mathbf{w}_{2}^{H} \mathbf{u} \mathbf{s}_{1,1}^{H} \mathbf{w}_{1}\right)}{\sqrt{M}} \\
\frac{\rho_{1} \sigma_{n}\left(\mathbf{w}_{1}^{H} \mathbf{u} \mathbf{s}_{1}^{H} \mathbf{w}_{2}+\mathbf{w}_{2}^{H} \mathbf{s}_{1} \mathbf{u}^{H} \mathbf{w}_{1}\right)}{\sqrt{M}} \\
\sqrt{M}
\end{gathered}
$$

As we minimize the power $\mathbf{w}^{H} \mathbf{R} \mathbf{w}$ subject to and $\mathbf{w}_{2}^{H} \mathbf{s}_{1}=$ 0 , the natural selection is that $\mathbf{w}_{1}$ be almost orthogonal to all the signals including $\mathbf{u}$ (except of course). As a result the order of $\mathbf{w}_{1}$ will not change and $\mathbf{w}_{1}^{H} \mathbf{R}_{1} \mathbf{s}_{1}=\left(\mathbf{s}_{1}^{H} \mathbf{R}_{1}^{-1} \mathbf{s}_{1}\right)^{-1} \approx$ $\sigma_{n}^{2} / N$ still holds. After assuming the orthogonality and substituting the above two constraints as well, we are left with the contributions $O\left(\rho_{1} \sigma_{n}\left(\mathbf{u}^{H} \mathbf{w}_{2}+\mathbf{w}_{2}^{H} \mathbf{u}\right) / \sqrt{M}\right), O\left(\rho_{1}^{2}\left(\beta_{1,1}^{*} \mathbf{s}_{1,1}^{H} \mathbf{w}_{2}+\right.\right.$ $\left.\left.\beta_{1,1} \mathbf{w}_{2}^{H} \mathbf{s}_{1,1}\right) / \sqrt{M}\right)$, and $\rho_{1}^{2} / \sqrt{M}$. Now ignoring the constant terms our minimization problem for obtaining an approximate highest order for $\mathbf{w}_{2}$ is equivalent to minimizing
$\mathbf{w}_{2}^{H} \mathbf{R}_{1} \mathbf{w}_{2}+O\left(\rho_{1} \sigma_{n}\left(\mathbf{u}^{H} \mathbf{w}_{2}+\mathbf{w}_{2}^{H} \mathbf{u}\right) / \sqrt{M}\right)$ subject to $\mathbf{w}_{2}^{H} \mathbf{s}_{1}=0$ or minimize $\mathbf{w}_{2}^{H} \mathbf{R}_{1} \mathbf{w}_{2}+O\left(\rho_{1}^{2}\left(\beta_{1,1}^{*} \mathbf{s}_{1,1}^{H} \mathbf{w}_{2}+\beta_{1,1} \mathbf{w}_{2}^{H} \mathbf{s}_{1,1}\right) / \sqrt{M}\right)$ subject to the same constraint. If the dominant term out of the last two terms is $O\left(\rho_{1} \sigma_{n}\left(\mathbf{u}^{H} \mathbf{w}_{2}+\mathbf{w}_{2}^{H} \mathbf{u}\right) / \sqrt{M}\right)$, then we have the same case as before but with $\mathbf{R}_{1}=\rho_{1}^{2}\left|\beta_{1,1}\right|^{2} \mathbf{s}_{1,1} \mathbf{s}_{1,1}^{H}+\sigma_{n}^{2} \mathbf{I}_{N}$. However, in this case, using a similar argument and using (A.10)-(A.14) we can prove that $T(n)_{n \neq n_{1,1}} \approx O(N / M)$ as follows.

The solution for this case would be

$$
\mathbf{w}_{2}=O\left(\frac{\rho_{1} \sigma_{n}}{\sqrt{M}}\right) \mathbf{R}_{1}^{-1} \mathbf{u}-\frac{\rho_{1} \sigma_{n}}{\sqrt{M}} \frac{\left(\mathbf{s}_{1}^{H} \mathbf{R}_{1}^{-1} \mathbf{u}\right)}{\left(\mathbf{s}_{1}^{H} \mathbf{R}_{1}^{-1} \mathbf{s}_{1}\right)} \mathbf{R}_{1}^{-1} \mathbf{s}_{1} .
$$

The first part of the above expression is simplified as follows (expanding $\mathbf{R}_{1}^{-1} \mathbf{u}$ ):

$$
\begin{aligned}
& \approx O \frac{\rho_{1} \sigma_{n}}{\sqrt{M}} \cdot \frac{1}{\sigma_{n}^{2}}\left(\mathbf{I}_{N}-\frac{\rho_{1}^{2}\left|\beta_{11}\right|^{2} \mathbf{s}_{1,1} \mathbf{s}_{1,1}^{H}}{\sigma_{n}^{2}+N \rho_{1}^{2}\left|\beta_{11}\right|^{2}}\right) \mathbf{u} \\
& \approx O \frac{\rho_{1}}{\sqrt{M}} \cdot \frac{1}{\sigma_{n}}\left(\mathbf{I}_{N}-\frac{\mathbf{s}_{1,1} \mathbf{s}_{1,1}^{H}}{N}\right) \mathbf{u} \quad\left(\text { for } N\left|\beta_{1,1}\right|^{2} \gg 1\right) \\
& \approx O\left(\frac{\rho_{1} \mathbf{u}}{\sigma_{n} \sqrt{M}}\right)-O\left(\frac{\rho_{1}}{\sigma_{n}} \frac{\mathbf{s}_{1,1}}{\sqrt{M}} \frac{\mathbf{s}_{1,1}^{H} \mathbf{u}}{N}\right) .
\end{aligned}
$$

The second part of the expression is expanded (using $\left.\mathbf{w}_{1}^{H} \mathbf{R}_{1} \mathbf{w}_{1}=\left(\mathbf{s}_{1}^{H} \mathbf{R}_{1}^{-1} \mathbf{s}_{1}\right)^{-1} \approx \sigma_{n}^{2} / N\right)$ as

$$
\begin{aligned}
O \frac{\rho_{1} \sigma_{n}}{\sqrt{M}} & \frac{\left(\mathbf{s}_{1}^{H} \mathbf{R}_{1}^{-1} \mathbf{u}\right)}{\left(\mathbf{s}_{1}^{H} \mathbf{R}_{1}^{-1} \mathbf{s}_{1}\right)} \mathbf{R}_{1}^{-1} \mathbf{s}_{1} \\
\approx & \frac{\rho_{1} \sigma_{n}}{\sqrt{M}}\left[\frac{\left(\mathbf{s}_{1}^{H} \mathbf{R}_{1}^{-1} \mathbf{u}\right) \sigma_{n}^{2}}{N}\right] \mathbf{R}_{1}^{-1} \mathbf{s}_{1} \\
\approx & O \frac{\rho_{1}^{2} \sigma_{n}^{3}}{\sqrt{M} N}\left[\frac{\mathbf{s}_{1}^{H}}{\sigma_{n}^{2}}\left(\mathbf{I}_{N}-\frac{\rho_{1}^{2}\left|\beta_{1,1}\right|^{2} \mathbf{s}_{1,1} \mathbf{s}_{1,1}^{H}}{\left(\sigma_{n}^{2}+N\left|\beta_{1,1}\right|^{2} \rho_{1}^{2}\right)}\right) \mathbf{u}\right] \\
& \times\left[\frac{1}{\sigma_{n}^{2}}\left(\mathbf{s}_{1}-\frac{\rho_{1}^{2}\left|\beta_{1,1}\right|^{2} \mathbf{s}_{1,1} \mathbf{s}_{1,1}^{H} \mathbf{s}_{1}}{\left(\sigma_{n}^{2}+N\left|\beta_{1,1}\right|^{2} \rho_{1}^{2}\right)}\right)\right] \quad \text { using }
\end{aligned}
$$

Now applying $N\left|\beta_{1,1}\right|^{2}$ snr $\gg 1$ (i.e., $\sigma_{n}^{2}+N\left|\beta_{1,1}\right|^{2} \rho_{1}^{2} \approx$ $N\left|\beta_{1,1}\right|^{2} \rho_{1}^{2}$ ) we have

$$
\begin{gathered}
\approx O \frac{\rho_{1}}{\sigma_{n} \sqrt{M} N}\left[\mathbf{s}_{1}^{H}\left(\mathbf{I}_{N}-\frac{\mathbf{s}_{1,1} \mathbf{s}_{1,1}^{H}}{N}\right) \mathbf{u}\left(\mathbf{s}_{1}-\frac{\mathbf{s}_{1,1} \mathbf{s}_{1,1}^{H} \mathbf{s}_{1}}{N}\right)\right] \\
\approx O \frac{\rho_{1}}{\sigma_{n} \sqrt{M} N}\left[\left(\mathbf{s}_{1}^{H} \mathbf{u}-\frac{\mathbf{s}_{1}^{H} \mathbf{s}_{1,1} \mathbf{s}_{1,1}^{H} \mathbf{u}}{N}\right)\left(\mathbf{s}_{1}-\frac{\mathbf{s}_{1,1} \mathbf{s}_{1,1}^{H} \mathbf{s}_{1}}{N}\right)\right] \\
\approx O \frac{\rho_{1}}{\sigma_{n}}\left[\frac{\left(\mathbf{s}_{1}^{H} \mathbf{u}\right)}{N} \frac{\mathbf{s}_{1}}{\sqrt{M}}-\frac{\left(\mathbf{s}_{1}^{H} \mathbf{u}\right)}{N} \frac{\mathbf{s}_{11}}{\sqrt{M}} \frac{\left(\mathbf{s}_{1,1}^{H} \mathbf{s}_{1}\right)}{N}\right. \\
-\frac{\left(\mathbf{s}_{1}^{H} \mathbf{s}_{1,1}\right)}{N} \frac{\left(\mathbf{s}_{1,1}^{H} \mathbf{u}\right)}{N} \frac{\mathbf{s}_{1}}{\sqrt{M}} \\
\left.+\frac{\left(\mathbf{s}_{1}^{H} \mathbf{s}_{1,1}\right)}{N} \frac{\left(\mathbf{s}_{1,1}^{H} \mathbf{u}\right)}{N} \frac{\mathbf{s}_{1,1}}{\sqrt{M}} \frac{\left(\mathbf{s}_{1,1}^{H} \mathbf{s}_{1}\right)}{N}\right] .
\end{gathered}
$$


When the two expressions are added ((A.56) and (A.58)), we can conclude that the dominant order term is $\approx$ $O\left(\rho_{1} \mathbf{u} / \sigma_{n} \sqrt{M}\right)$.

On the other hand suppose the dominant contribution is $O\left(\rho_{1}^{2}\left(\beta_{1,1}^{*} \mathbf{w}_{1}^{H} \mathbf{s}_{1} \mathbf{s}_{1,1}^{H} \mathbf{w}_{2}+\beta_{1,1} \mathbf{w}_{2}^{H} \mathbf{s}_{1,1} \mathbf{s}_{1}^{H} \mathbf{w}_{1}\right) / \sqrt{M}\right)$ term then we have to minimize $P_{\text {out }}=\mathbf{w}_{1}^{H} \mathbf{R}_{x} \mathbf{w}_{1}+\mathbf{w}_{2}^{H} \mathbf{R}_{x} \mathbf{w}_{2}+\mathbf{w}_{1}^{H} \mathbf{Q}^{H} \mathbf{w}_{2}+$ $\mathbf{w}_{2}^{H} \mathbf{Q} \mathbf{w}_{1}$, which after substituting the constraints and noting that $\mathbf{R}_{1}=\rho_{1}^{2}\left|\beta_{1,1}\right|^{2} \mathbf{s}_{1,1} \mathbf{s}_{1,1}^{H}+\sigma_{n}^{2} \mathbf{I}_{N}, \mathbf{R}_{x}=\mathbf{R}_{1}+\rho_{1}^{2} \mathbf{s}_{1} \mathbf{s}_{1}^{H}$ is reduced to minimizing $P_{\text {out }}=\rho_{1}^{2}+\mathbf{w}_{1}^{H} \mathbf{R}_{1} \mathbf{w}_{1}+\mathbf{w}_{2}^{H} \mathbf{R}_{1} \mathbf{w}_{2}+$ $O\left(\rho_{1}^{2}\left(\beta_{1,1}^{*} \mathbf{s}_{1,1}^{H} \mathbf{w}_{2}+\beta_{1,1} \mathbf{w}_{2}^{H} \mathbf{s}_{1,1}\right) / \sqrt{M}\right)$ subject to the constraints: $\mathbf{w}_{1}^{H} \mathbf{s}_{1}=1$ and $\mathbf{w}_{2}^{H} \mathbf{s}_{1}=0$. This problem has been solved earlier without the factor $O(1 / \sqrt{M})$ and as a result we can write (using (A.4))

$$
\begin{gathered}
\mathbf{w}_{2}=-O\left(\frac{\beta_{11} \rho_{1}^{2}}{\sqrt{M}}\right) \mathbf{R}_{1}^{-1} \mathbf{s}_{1,1}+O\left(\frac{\beta_{11} \rho_{1}^{2}}{\sqrt{M}}\right)\left(\frac{\mathbf{s}_{1}^{H} \mathbf{R}_{1}^{-1} \mathbf{s}_{1,1}}{\mathbf{s}_{1}^{H} \mathbf{R}_{1}^{-1} \mathbf{s}_{1}}\right) \mathbf{R}_{1}^{-1} \mathbf{s}_{1}, \\
\mathbf{w}_{1}=\frac{\mathbf{R}_{1}^{-1} \mathbf{s}_{1}}{\left(\mathbf{s}_{1}^{H} \mathbf{R}_{1}^{-1} \mathbf{s}_{1}\right)} .
\end{gathered}
$$

Now applying earlier results we can show that (A.23), (A.27) $\left\|\mathbf{w}_{1}\right\|^{2} \approx 1 / N$ and $\left\|\mathbf{w}_{2}\right\|^{2} \approx 1 /\left(N M\left|\beta_{1,1}\right|^{2}\right)$ for $N\left|\beta_{1,1}\right|^{2}$ snr $\gg$ 1 and furthermore

$$
\begin{aligned}
P_{\text {out }} \approx & \rho_{1}^{2}+\mathbf{w}_{1}^{H} \mathbf{R}_{1} \mathbf{w}+\left.\sigma_{n}^{2}|| \mathbf{w}_{2}\right|^{2}+\rho_{1}^{2}\left|\beta_{1,1}\right|^{2}\left|\mathbf{w}_{2}^{H} \mathbf{s}_{1,1}\right|^{2} \\
& +O\left(\frac{\rho_{1}^{2}\left(\beta_{1,1}^{*} \mathbf{s}_{1,1}^{H} \mathbf{w}_{2}+\beta_{1,1} \mathbf{w}_{2}^{H} \mathbf{s}_{1,1}\right)}{\sqrt{M}}\right) \\
\approx & \sigma_{n}^{2}\|\mathbf{w}\|^{2}+\rho_{1}^{2}+\rho_{1}^{2}\left|\beta_{1,1}\right|^{2}\left|\mathbf{w}_{2}^{H} \mathbf{s}_{1,1}\right|^{2} \\
& +O\left(\frac{\rho_{1}^{2}\left(\beta_{1,1}^{*} \mathbf{s}_{1,1}^{H} \mathbf{w}_{2}+\beta_{1,1} \mathbf{w}_{2}^{H} \mathbf{s}_{1,1}\right)}{\sqrt{M}}\right)
\end{aligned}
$$

(Note: $\mathbf{w}_{1}^{H} \mathbf{s}_{1,1} \approx 0$ has been assumed).

Now we have

$$
\begin{aligned}
\beta_{1,1} \mathbf{w}_{2}^{H} \mathbf{s}_{1,1}=O( & -\frac{\left|\beta_{1,1}\right|^{2}}{\sqrt{M}} \rho_{1}^{2} \mathbf{s}_{1,1}^{H} \mathbf{R}_{1}^{-1} \mathbf{s}_{1,1} \\
& \left.+\frac{\left|\beta_{1,1}\right|^{2}}{\sqrt{M}} \rho_{1}^{2} \frac{\left|\mathbf{s}_{1}^{H} \mathbf{R}_{1}^{-1} \mathbf{s}_{1,1}\right|^{2}}{\mathbf{s}_{1}^{H} \mathbf{R}_{1}^{-1} \mathbf{s}_{1}}\right),
\end{aligned}
$$

using the earlier results (i.e., $\left|\mathbf{s}_{1}^{H} \mathbf{R}_{1}^{-1} \mathbf{s}_{1,1}\right| / \mathbf{s}_{1}^{H} \mathbf{R}_{1}^{-1} \mathbf{s}_{1} \approx 0$ (A.19)) we have(using (A.12))

$$
\begin{aligned}
\beta_{1,1} \mathbf{w}_{2}^{H} \mathbf{s}_{1,1} & \approx-\frac{\left|\beta_{1,1}\right|^{2} \rho_{1}^{2} N}{\sqrt{M}\left(\sigma_{n}^{2}+N\left|\beta_{1,1}\right|^{2} \rho_{1}^{2}\right)} \\
& =\frac{\left|\beta_{1,1}\right|^{2} N \text { snr }}{\sqrt{M}\left(1+N\left|\beta_{1,1}\right|^{2} \mathrm{snr}\right)} \\
& \approx-\frac{1}{\sqrt{M}}
\end{aligned}
$$

$\left(\right.$ for $\left.N\left|\beta_{1,1}\right|^{2} \gg 1\right)$.
Now substituting, $O\left(\rho_{1}^{2}\left(\beta_{1,1}^{*} \mathbf{s}_{1,1}^{H} \mathbf{w}_{2}+\beta_{1,1} \mathbf{w}_{2}^{H} \mathbf{s}_{1,1}\right) / \sqrt{M}\right) \approx$ $O\left(\rho_{1}^{2} / M\right), \mathbf{w}_{1}^{H} \mathbf{R}_{1}^{-1} \mathbf{w}_{1}=\left(\mathbf{s}_{1}^{H} \mathbf{R}_{1}^{-1} \mathbf{s}_{1}\right)^{-1} \approx \sigma_{n}^{2} / N,\left\|\mathbf{w}_{2}\right\|^{2} \approx$ $1 /\left(N M\left|\beta_{1,1}\right|^{2}\right)$ and the above result in the above expression for $P_{\text {out }}$ and further simplifying we arrive at

$$
\begin{array}{r}
P_{\text {out }} \approx \rho_{1}^{2}+\sigma_{n}^{2}\left(\frac{1}{N}\right)+O\left(\frac{\sigma_{n}^{2}}{\left(M N\left|\beta_{1,1}\right|^{2}\right)}\right)+O\left(\frac{\rho_{1}^{2}}{M}\right) \\
\text { for } N\left|\beta_{1,1}\right|^{2} \gg 1,
\end{array}
$$

since the term $O\left(1 /\left(M N\left|\beta_{1,1}\right|^{2}\right)\right)$ is very small compared to the orders of all other terms we end up with $P_{\text {out }} \approx \rho_{1}^{2}+$ $\sigma_{n}^{2}(1 / N)+O\left(\rho_{1}^{2} / M\right)$ for $N\left|\beta_{1,1}\right|^{2} \gg 1$.

Now substituting this result in (6) we have

$$
T_{s}(n)_{n \neq n_{1,1}} \approx \frac{\left(\mathbf{s}_{1} \mathbf{R}_{1}^{-1} \mathbf{s}_{1}\right)^{-1}+\rho_{1}^{2}}{\rho_{1}^{2}+\sigma_{n}^{2}(1 / N)+O\left(\rho_{1}^{2} /(M)\right)}-1 .
$$

This is equivalent to

$$
T_{s}(n)_{n \neq n_{1,1}} \approx O\left(\frac{1}{M}\right)
$$

which produces much small noise floor and hence this option is discarded in estimating the signal processing gain.

The final expression for the signal processing gain of the lag finder is obtained by the use of the earlier result as

$$
\frac{T_{s}(n)_{n=n_{1,1}}}{T_{s}(n)_{n \neq n}} \approx O\left(\frac{N\left|\beta_{1,1}\right|^{2} \mathrm{snr}}{(N / M)}\right) \approx M\left|\beta_{1,1}\right|^{2} \text { snr. }
$$

\section{B.}

Lemma B. Suppose the square matrix $\mathbf{A}$ is added to an additional dyad term $\mathbf{u u}^{H}$, where $\mathbf{u}$ is a column vector, then the inversion of the new matrix is given by (e.g., [10, Van Trees, page 1348])

$$
\left(\mathbf{A}+\mathbf{u} \mathbf{u}^{H}\right)^{-1}=\mathbf{A}^{-1}-\frac{\mathbf{A}^{-1} \mathbf{u} \mathbf{u}^{H} \mathbf{A}^{-1}}{1+\mathbf{u}^{H} \mathbf{A}^{-1} \mathbf{u}} .
$$

By definition one have $\mathbf{R}_{x}=\rho_{1}^{2} \mathbf{s}_{1} \mathbf{s}_{1}^{H}+\mathbf{R}_{1}$. Applying the above lemma one have the following identity:

$$
\mathbf{R}_{x}^{-1}=\mathbf{R}_{1}^{-1}-\frac{\rho_{1}^{2}\left(\mathbf{R}_{1}^{-1} \mathbf{s}_{1} \mathbf{s}_{1}^{H} \mathbf{R}_{1}^{-1}\right)}{1+\rho_{1}^{2}\left(\mathbf{s}_{1}^{H} \mathbf{R}_{1}^{-1} \mathbf{s}_{1}\right)} .
$$

This leads to the expression

$$
\begin{gathered}
\mathbf{s}_{1}^{H} \mathbf{R}_{x}^{-1} \mathbf{s}_{1}=\mathbf{s}_{1}^{H} \mathbf{R}_{1}^{-1} \mathbf{s}_{1}-\frac{\rho_{1}^{2}\left(\mathbf{s}_{1}^{H} \mathbf{R}_{1}^{-1} \mathbf{s}_{1} \mathbf{s}_{1}^{H} \mathbf{R}_{1}^{-1} \mathbf{s}_{1}\right)}{1+\rho_{1}^{2}\left(\mathbf{s}_{1}^{H} \mathbf{R}_{1}^{-1} \mathbf{s}_{1}\right)} \\
=\frac{\mathbf{s}_{1}^{H} \mathbf{R}_{1}^{-1} \mathbf{s}_{1}}{1+\rho_{1}^{2}\left(\mathbf{s}_{1}^{H} \mathbf{R}_{1}^{-1} \mathbf{s}_{1}\right)} \\
\left(\mathbf{s}_{1}^{H} \mathbf{R}_{x}^{-1} \mathbf{s}_{1}\right)^{-1}=\rho_{1}^{2}+\left(\mathbf{s}_{1}^{H} \mathbf{R}_{1}^{-1} \mathbf{s}_{1}\right)^{-1} .
\end{gathered}
$$

\section{Acknowledgments}

Authors would like to thank the Defence Science and Technology Organisation for sponsoring this work. Also they would like to thank the referees for the valuable comments and suggestions. 


\section{References}

[1] P. E. Howland, "A passive metric radar using the transmitters of opportunity," in Proceedings of IEEE International Conference on Radar, pp. 251-256, Paris, France, May 1994.

[2] P. E. Howland, "Target tracking using television-based bistatic radar," IEE Proceedings: Radar, Sonar and Navigation, vol. 146, no. 3, pp. 166-174, 1999.

[3] P. E. Howland, D. Maksimiuk, and G. Reitsma, "FM radio based bistatic radar," IEE Proceedings: Radar, Sonar and Navigation, vol. 152, no. 3, pp. 107-115, 2005.

[4] H. D. Griffiths, C. J. Baker, J. Baubert, N. Kitchen, and M. Treagust, "Bistatic radar using satellite-borne illuminators of opportunity," in Proceedings of IEEE International Conference on Radar, pp. 1-5, Edinburgh, UK, October 2002.

[5] K. S. Kulpa and Z. Czekała, "Masking effect and its removal in PCL radar," IEE Proceedings: Radar, Sonar and Navigation, vol. 152, no. 3, pp. 174-178, 2005.

[6] W. Lewandowski and C. Thomas, "GPS time transfer," Proceedings of the IEEE, vol. 79, no. 7, pp. 991-1000, 1991.

[7] D. Kirchner, "Two-way time transfer via communication satellites," Proceedings of the IEEE, vol. 79, no. 7, pp. 983-990, 1991.

[8] N. J. Willis, Bistatic Radar, Artech House, Boston, Mass, USA, 1991.

[9] J. C. Kirk Jr., "Bistatic SAR Motion Compensation," in Proceedings of the International Radar Conference, pp. 360-365, Arlington, Va, USA, May 1985.

[10] S. M. Kogon, E. J. Holder, and D. B. Williams, "Mainbeam jammer suppression using multipath returns," in Proceedings of the 31st Asilomar Conference on Signals, Systems and Computers, vol. 1, pp. 279-283, Pacific Grove, Calif, USA, November 1997.

[11] Y. Seliktar, E. J. Holder, and D. B. Williams, "An adaptive monopulse processor for angle estimation in a mainbeam jamming and coherent interference scenario," in Proceedings of IEEE International Conference on Acoustics, Speech, and Signal Processing (ICASSP '98), vol. 4, pp. 2037-2040, Seattle, Wash, USA, May 1998.

[12] S. M. Kogon, D. B. Williams, and E. J. Holder, "Exploiting coherent multipath for mainbeam jammer suppression," IEE Proceedings: Radar, Sonar and Navigation, vol. 145, no. 5, pp. 303-308, 1998.

[13] R. L. Fante and J. A. Torres, "Cancellation of diffuse jammer multipath by an airborne adaptive radar," IEEE Transactions on Aerospace and Electronic Systems, vol. 31, no. 2, pp. 805820, 1995.

[14] D. Madurasinghe and A. P. Shaw, "Mainlobe jammer nulling via TSI finders: a space fast-time adaptive processor," EURASIP Journal on Applied Signal Processing, vol. 2006, Article ID 48789, 13 pages, 2006.

[15] H. L. Van Trees, Optimum Array Processing, Part IV of Detection Estimation, and Modulation Theory, John Wiley \& Sons, New York, NY, USA, 2002.

[16] R. O. Schmidt, "Multiple emitter location and signal parameter estimation," in Proceedings of the RADC Spectrum Estimation Workshop, pp. 243-258, Rome Air Development Centre, Rome, NY, USA, October 1979, RADC-TR-79-63.

[17] R. O. Schmidt, "Multiple emitter location and signal parameter estimation," IEEE Transactions on Antennas and Propagation, vol. 34, no. 3, pp. 276-280, 1986. 